LASTRE
Laboratoire d'Applications des Systèmes Tychastiques
Régulés
R
S

\title{
Guaranteed Inertia Functions in Dynamical Games
}

\author{
Jean-Pierre Aubin and Patrick Saint-Pierre \\ LASTRE (Laboratoire d'Applications des Systèmes Tychastiques Régulés ) \\ and Centre de Recherche Viabilité, Jeux, Contrôle \\ Université Paris-Dauphine
}

Draft of March 23, 2006

\begin{abstract}
This paper deals with inertia functions in control theory introduced in [7, 8, Aubin, Bernardo $\&$ Saint-Pierre] and their adaptation to dynamical games. The inertia function associates with any initial state-control pair the smallest of the worst norms over time of the velocities of the controls regulating viable evolutions. For tychastic systems (parameterized systems where the parameters are tyches, disturbances, perturbations, etc.), the palicinesia of a tyche measure the worst norm over time of the velocities of the tyches. The palicinesia function is the largest palicinesia threshold c such that all evolutions with palicinesia smaller than or equal to $c$ are viable. For dynamical games where one parameter is the control and the other one is a tyche (games against nature or robust control), we define the guaranteed inertia function associated with any initial state-control-tyche triple the best of the worst of the norms of the velocities of the controls and of the tyches and study their properties. Viability Characterizations and Hamilton-Jacobi equations of which these inertia and palicinesia functions are solutions are provided.
\end{abstract}




\section{Introduction}

Let us denote by $\mathcal{P}(x, u)$ the set of solutions to a control system

$$
\begin{cases}(i) & x^{\prime}(t):=f(x(t), u(t)) \\ (i i) & u(t) \in U(x(t))\end{cases}
$$

starting at $(x, u)$. Its inertia function $\alpha$ is defined by

$$
\alpha(x, u):=\inf _{(x(\cdot), u(\cdot)) \in \mathcal{P}(x, u)} \sup _{t \geq 0}\left\|u^{\prime}(t)\right\|
$$

This is a very useful function for studying special evolutions, such as heavy evolutions introduced in [11, Aubin \& Frankowska], which minimize at each instant the norm of the velocities of the controls. The inertia function is the prototype of value functions of special type of optimal control problems original in two ways: (i) The intertemporal optimality criterium bears on the velocities of the controls (and not only on the evolutions of the states and the controls, as it is usually the case); (ii) The criterion is not as usual an integral criterion, but a supremum over time of a Lagrangian function (here, the norm).

However, we prove that its epigraph is the viability kernel of an auxiliary set under an auxiliary system, called the associated metasystem. This allows us to use the Viability Kernel Algorithm to compute it and to translate in this framework the properties of viability kernels. Among them, the tangential characterizations of viability kernels imply that the inertia function is a solution to an adequate Hamilton-Jacobi equation.

When the parameter $u$ is no longer a control, but a tyche (the Greek name for chance), or a disturbance, a perturbation, as in robust control, the worst norm $\sup _{t>0}\left\|u^{\prime}(t)\right\|$ over time of the velocities of the tyches, a dynamical measure of risk, is called its palicinesia (from Greek, palin $=$ rapid change, kinesis $=$ velocity). In this framework, the question we ask is to find the largest palicinesia threshold $c$ such that all evolutions governed by $x^{\prime}(t)=f(x(t), u(t))$ with palicinesia bounded by $c$ and starting from $(c, u)$ satisfy the constraints $u(t) \in U(x(t))$. These largest palicinesia thresholds define the palicinesia function.

In the case of dynamical games where one parameter is a control and the other one a tyche, the guaranteed inertia function associates with any initial state-control-tyche triple the best of the worst of the norms of the velocities of the controls and of the tyches.

The inertia and palicinesia functions are respectively the smallest and the largest nonnegative (generalized in the generalized sense of Barron-Jensen \& Frankowska, 13, Barron \& Jensen], 9, 26, 27, Frankowska]) solutions to Hamilton-Jacobi-Bellman partial differential equation

$$
\left\langle\frac{\partial \mathbf{v}(x, u)}{\partial x}, f(x, u)\right\rangle-\mathbf{v}(x, u)\left\|\frac{\partial \mathbf{v}(x, u)}{\partial u}\right\|_{\star}=\sum_{i=1}^{n} \frac{\partial \mathbf{v}(x, u)}{\partial x_{i}} f_{i}(x, u)-\mathbf{v}(x, u)\left\|\frac{\partial \mathbf{v}(x, u)}{\partial u}\right\|_{\star}=0
$$


on the graph of $U$, where $\|p\|_{\star}:=\sup _{\|v\| \leq 1}\langle p, v\rangle$ denotes the dual norm.

The guaranteed inertia function is the largest nonnegative solution to Hamilton-JacobiIsaacs equation

$$
\left\langle\frac{\partial \mathbf{w}(x, u, v)}{\partial x}, f(x, v)\right\rangle+\mathbf{w}(x, u, v)\left\|\frac{\partial \mathbf{w}(x, u, v)}{\partial u}\right\|_{\star}-\mathbf{w}(x, u, v)\left\|\frac{\partial \mathbf{w}(x, u, v)}{\partial v}\right\|_{\star}=0
$$

The paper is organized as follows: Section 2 recalls the needed property of viability kernels and section 3 studies inertia functions. Two examples of inertia functions are provided: In section 4 , the inertia function of the basic equation of population dynamics and in section 5 , an example of management of renewable resources. Section 6 defines tychastic and recalls the main characterization of invariance kernels, whereas section 7 shows that stochastic viability kernels are particular cases of invariance kernels for an associated tychastic system. Section 8 is devoted to palicinesia functions. Section 9 introduces the guaranteed viability kernel under a dynamical game and the results recalled is applied to study the guaranteed inertia function.

\section{Control Systems}

We denote by $X$ the state space, assumed to be a finite dimensional vector space. The space of controls is another finite dimensional vector space $\mathcal{U}$.

\section{Definition 2.1 We introduce}

1. $f: X \times \mathcal{U} \mapsto X$, a map associating the velocity $f(x, u)$ of the state $x$ with any statecontrol pair $(x, u)$,

2. the cybernetic map $U: X \leadsto \mathcal{U}$, a set-valued map associating a set $U(x)$ of controls feeding back on the state $x$.

The evolutionary system $\mathcal{S}: X \leadsto \mathcal{C}(0, \infty ; X)$ defined by the control system $(f, U)$ is the setvalued map associating with any $x \in X$ the set $\mathcal{S}(x)$ of evolutions $x(\cdot)$ governed by the control (or regulated) system

$$
\begin{cases}(i) & x^{\prime}(t)=f(x(t), u(t)) \\ (i i) & u(t) \in U(x(t))\end{cases}
$$

starting from $x$.

When parameters are controls, we are interested in regulating the system in the sense that we are looking for at least one evolution of the evolutionary system satisfying an 


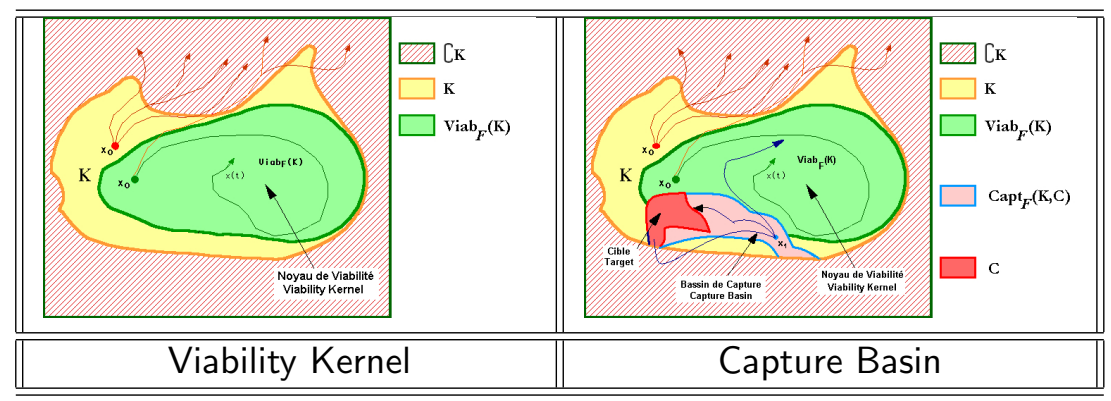

Figure 1: Viability Kernels and Capture Basins. The environment (constrained set) is yellow, the target red. The viability kernel in green and the capture basin in light pink.

evolutionary property, one can regard the evolutionary system as a control system or a cybernetic system. We select among such evolutionary properties the viability property of an environment and the capturability property of a target:

Definition 2.2 Let $K \subset X$ be a constrained set and $C \subset K$ be a target.

1. The subset $\operatorname{Viab}_{\mathcal{S}}(K, C)$ of initial states $x_{0} \in K$ such that at least one evolution $x(\cdot) \in \mathcal{S}\left(x_{0}\right)$ starting at $x_{0}$ is viable in $K$ for all $t \geq 0$ or viable in $K$ until it reaches $C$ in finite time is called the viability kernel of $K$ with target $C$ under $\mathcal{S}$.

When the target $C=\emptyset$ is the empty set, we say that $\operatorname{Viab}_{\mathcal{S}}(K):=\operatorname{Viab}_{\mathcal{S}}(K, \emptyset)$ is the viability kernel of $K$.

2. The subset $\operatorname{Capt}_{\mathcal{S}}(K, C)$ of initial states $x_{0} \in K$ such that at least one evolution $x(\cdot) \in \mathcal{S}\left(x_{0}\right)$ starting at $x_{0}$ is viable in $K$ until it reaches $C$ in finite time is called the capture basin of $C$ viable in $K$ under $\mathcal{S}$. When $K=X$ is the whole space, we say that $\operatorname{Capt}_{\mathcal{S}}(C):=\operatorname{Capt}_{\mathcal{S}}(X, C)$ is the capture basin of $C$.

We say that

1. a subset $K$ is viable under $\mathcal{S}$ if $K=\operatorname{Viab}_{\mathcal{S}}(K)$,

2. $K$ is viable outside the target $C \subset K$ under the evolutionary system $\mathcal{S}$ if $K=\operatorname{Viab}_{\mathcal{S}}(K, C)$,

3. $C$ is isolated in $K$ if $C=\operatorname{Viab}_{\mathcal{S}}(K, C)$,

4. $K$ is a repeller if $\operatorname{Viab}_{\mathcal{S}}(K)=\emptyset$, i.e., if the empty set is isolated in $K$.

It is easy to check that whenever the complement $K \backslash C:=K \cap \complement C$ of the target $C$ in the environment is a repeller, the viability kernel with target $C$ and the capture basin of target $C$ coincide. This will be the case of the auxiliary systems introduced later for deriving the announced results.

We deduce from [9, Aubin \& Catté] the following characterization of viability kernels: 
Theorem 2.3 The viability kernel $\operatorname{Viab}_{\mathcal{S}}(K, C)$ of $K$ outside the target $C$ is the unique subset between $C$ and $K$ that is both

1. viable outside $C$ (and is the largest subset $D \subset K$ viable outside $C$ ),

2. isolated in $K$ (and is the smallest subset $D \supset C$ isolated in $K$ ):

$$
\operatorname{Viab}_{\mathcal{S}}\left(K, \operatorname{Viab}_{\mathcal{S}}(K, C)\right)=\operatorname{Viab}_{\mathcal{S}}(K, C)=\operatorname{Viab}_{\mathcal{S}}\left(\operatorname{Viab}_{\mathcal{S}}(K, C), C\right)
$$

and thus, the unique bilateral fixed point $D$ between $C$ and $K$ in the sense that

$$
D=\operatorname{Viab}_{\mathcal{S}}(K, D)=\operatorname{Viab}_{\mathcal{S}}(D, C)
$$

The same properties are shared by the capture basins.

The Viability and Invariance Theorems characterize capture basins through tangential conditions involving tangent cones (contingent cone) to a subset $K$ at a point $x \in K$, introduced in the early 1930's independently by Bouligand and Severi : They adapt to any subset the concept of tangent space to manifolds: A direction $v \in X$ belongs to $T_{K}(x)$ if there exist sequences $h_{n}>0$ and $v_{n} \in X$ converging to 0 and $v$ respectively such that

$$
\forall n \geq 0, \quad x+h_{n} v_{n} \in K
$$

Let $x(\cdot): t \in \mathbb{R}_{+} \mapsto x(t) \in X$ be a differentiable evolution viable in $K$ on an open interval $\mathbb{I}$ : $\forall t \in \mathbb{I}, x(t) \in K$. Then

$$
\forall t \in \mathbb{I}, \quad x^{\prime}(t) \in T_{K}(x(t))
$$

We summarize the main characterization theorem of viability kernel in terms of tangent cones (see [4, 5, Aubin] and the forthcoming monograph [6, Aubin, Bayen, Bonneuil \& SaintPierre] for more details). Recall that a set-valued map $F$ is Marchaud if

$$
\begin{cases}(i) & \text { the graph and the domain of } F \text { are nonempty and closed } \\ (\text { ii }) & \text { the values } F(x) \text { of } F \text { are convex } \\ (\text { iii }) & \exists c>0 \text { such that } \forall x \in X \\ & \|F(x)\|:=\sup _{v \in F(x)}\|v\| \leq c(\|x\|+1)\end{cases}
$$

Theorem 2.4 Assume that the dynamics $f$ is continuous with linear growth and affine with respect to the controls and the cybernetic map $U$ is Marchaud. Assume also that the target $C$ and the environment $K$ are closed. Then the viability kernel $\operatorname{Viab}_{\mathcal{S}}(K, C)$ is the largest closed subset $D$ between $C$ and $K$ satisfying the tangential condition

$$
\forall x \in D \backslash C, \exists u \in U(x) \text { such that } f(x, u) \in T_{D}(x)
$$


and

$$
\operatorname{Viab}_{\mathcal{S}}\left(K \backslash \operatorname{Viab}_{\mathcal{S}}(K, C)\right)=\emptyset
$$

The latter condition is automatically satisfied when $K \backslash C$ is a repeller.

If we assume furthermore that the dynamics $f$ and the map $U$ are Lipschitz, then the viability kernel $\operatorname{Viab}_{\mathcal{S}}(K, C)$ is the unique closed subset $D$ between $C$ and $K$ satisfying the Frankowska tangential conditions

$$
\left\{\begin{array}{l}
\text { (i) } \quad \forall x \in D \backslash C, \exists u \in U(x) \text { such that } f(x, u) \in \overline{\mathrm{co}}\left(T_{D}(x)\right) \\
(\text { ii }) \quad \forall x \in D \cap \operatorname{Int}(K), \forall u \in U(x),-f(x, u) \in \overline{\mathrm{co}}\left(T_{D}(x)\right) \\
(\text { iii }) \quad \forall x \in D \cap \partial K, \quad \forall u \in U(x),-f(x, u) \in \overline{\mathrm{CO}}\left(T_{D}(x)\right) \cup T_{\overline{\mathrm{C} K}}(x)
\end{array}\right.
$$

and condition (5).

\section{Inertia Functions}

We integrate now the definition of the environment $K$ in the definition of the cybernetic map $U$ by setting $U(x)=\emptyset$ whenever $x \notin K$. We denote by $\mathcal{P}(x, u)$ the set of solutions $(x(\cdot), u(\cdot))$ to the above parameterized system (1) starting at $(x, u)$.

Definition 3.1 The inertia function $\alpha$ of system (1) defined by

$$
\alpha(x, u):=\inf _{(x(\cdot), u(\cdot)) \in \mathcal{P}(x, u)} \sup _{t \geq 0}\left\|u^{\prime}(t)\right\| \in[0,+\infty]
$$

on $\operatorname{Graph}(U)$ associates any state-control pair $(x, u) \in \mathrm{Graph}(U)$ the minimal worst intertemporal inertia $\alpha(x, u)$ of the evolutions starting from $(x, u) \in \operatorname{Graph}(U)$.

From now on, we shall assume that $f$ is continuous and that the graph of $U$ is closed.

\subsection{Viability Characterization of Inertia Functions}

We shall characterize the inertia function in terms of the viability kernel of $\operatorname{Graph}(U) \times \mathbb{R}_{+}$ under the auxiliary system

$$
\begin{cases}(i) & x^{\prime}(t)=f(x(t), u(t)) \\ (i i) & u^{\prime}(t)=u_{1}(t) \\ (i i i) & y^{\prime}(t)=0 \\ & \text { where }\left\|u_{1}(t)\right\| \leq y(t)\end{cases}
$$

that we shall call the metasystem associated with initial control system (1). It is regulated by the velocities of the controls of initial system (1), called metacontrols. 
Theorem 3.2 The inertia function is related to the viability kernel of $\operatorname{Graph}(U) \times \mathbb{R}_{+}$under metasystem (7) by formula

$$
\alpha(x, u)=\inf _{(x, u, y) \in \operatorname{Viab}_{\mathbf{7}}\left(\operatorname{Graph}(U) \times \mathbb{R}_{+}\right)} y
$$

Proof - Indeed, to say that $(x, u, y)$ belongs to $\operatorname{Viab} \sqrt{7 p}\left(\operatorname{Graph}(U) \times \mathbb{R}_{+}\right)$amounts to saying that there exists an evolution $t \mapsto(x(t), u(t))$ governed by $(7)$ where $t \mapsto(x(t), u(t), y(t))$ is governed by control system (1) and where $y(t) \equiv y$. In other words, the solution $(x(\cdot), u(\cdot)) \in$ $\mathcal{P}(x, u)$ satisfies

$$
\forall t \geq 0, \quad\left\|u^{\prime}(t)\right\| \leq y
$$

so that $\alpha(x, u) \leq \sup _{t \geq 0}\left\|u^{\prime}(t)\right\| \leq y$.

Conversely, if $\alpha(x, u)<+\infty$, we can associate with any $\varepsilon>0$ an evolution $\left(x_{\varepsilon}(\cdot), u_{\varepsilon}(\cdot)\right) \in$ $\mathcal{P}(x, u)$ such that

$$
\forall t \geq 0,\left\|u_{\varepsilon}^{\prime}(t)\right\| \leq \alpha(x, u)+\varepsilon=: y_{\varepsilon}
$$

Therefore, setting $u_{\varepsilon_{1}}(t):=u_{\varepsilon}^{\prime}(t)$ and $y_{\varepsilon}(t)=y_{\varepsilon}$, we observe that $t \mapsto\left(x_{\varepsilon}(t), u_{\varepsilon}(t), y_{\varepsilon}\right)$ is a solution to the auxiliary system (7) viable in $\operatorname{Graph}(U) \times \mathbb{R}_{+}$, and thus, that $\left(x, u, y_{\varepsilon}\right)$ belongs to $\operatorname{Viab}_{77}\left(\operatorname{Graph}(U) \times \mathbb{R}_{+}\right)$. Hence

$$
\inf _{(x, u, y) \in \operatorname{Viab}_{\mathbf{7}}\left(\operatorname{Graph}(U) \times \mathbb{R}_{+}\right)} y \leq y_{\varepsilon}:=\alpha(x, u)+\varepsilon
$$

and it is enough to let $\varepsilon$ converge to 0 .

The metasystem (7) is Marchaud whenever the single-valued map $f$ is continuous, Lipschitz whenever the single-valued map $f$ is Lipschitz and the metaenvironement is closed whenever the graph of $U$ is closed and a closed convex process if $f$ is affine and the graph of $U$ is closed and convex. Hence it inherits of the properties of Marchaud, Lipschitz and convex systems respectively.

Theorem 3.3 If $f$ is continuous and the graph of $U$ is closed, the inertia function $\alpha$ is lower semicontinuous. Furthermore, from any $(x, u) \in \operatorname{Dom}(\alpha)$ starts at least one evolution $(\bar{x}(\cdot), \bar{u}(\cdot)) \in \mathcal{P}(x, u)$ such that

$$
\alpha(x, u)=\sup _{t \geq 0}\left\|\bar{u}^{\prime}(t)\right\|
$$

Il furthermore $f$ is affine and the graph of $U$ is closed and convex, then the inertia function is convex. 
Proof - Since the auxiliary system (7) is Marchaud and $f$ continuous and since the auxiliary environment $\operatorname{Graph}(U) \times \mathbb{R}_{+}$is closed by assumption, then the viability kernel $\operatorname{Viab}{ }_{77}\left(\operatorname{Graph}(U) \times \mathbb{R}_{+}\right)$is also closed. Let us consider a minimizing sequence

$$
\forall t \geq 0,\left\|u_{\varepsilon}^{\prime}(t)\right\| \leq \alpha(x, u)+\varepsilon=: y_{\varepsilon}
$$

and the associated evolution $t \mapsto\left(x_{\varepsilon}(t), u_{\varepsilon}(t), y_{\varepsilon}\right)$ which is a solution to the auxiliary system (7) viable in $\operatorname{Graph}(U) \times \mathbb{R}_{+}$. The upper semi-compactness of the associated evolutionary system implies that a subsequence (again denoted by) of $\left(x_{\varepsilon}(\cdot), u_{\varepsilon}(\cdot), y_{\varepsilon}\right)$ ) converges to a solution $(\bar{x}(\cdot), \bar{u}(\cdot), \alpha(x, u))$ satisfying

$$
\forall t \geq 0, \quad\left\|\bar{u}^{\prime}(t)\right\| \leq \alpha(x, u)
$$

Therefore, the infimum of the inertia function is achieved.

We associate with the inertia function in terms the useful concepts of inert retroaction map and crisis map:

Definition 3.4 We associate with the inertia function $\alpha$ the following set valued maps:

1. Inert Regulation Map $(c, x) \leadsto R(c ; x):=\{u \in \mathbb{R}$ such that $\alpha(x, u) \leq c\}$

2. Crisis Map $(c, u) \leadsto \Xi(c ; u):=\{x \in[a, b]$ such that $\alpha(x, u)=c\}$

When $c>0$, the subset $\Xi(c, u)$ is called the crisis zone of the control $u$ bounded by inertia threshold $c>0$.

When $c=0$, the subset $\Xi(0, u)$ is called the viability niche of the control $u$.

\subsection{Hamilton-Jacobi Equations for Inertia Functions}

One can prove that the inertia function $\alpha$ is is the smallest nonnegative lower semicontinuous solution to the Hamilton-Jacobi-Bellman partial differential equation: $\forall(x, u) \in$ $\operatorname{Graph}(U)$,

$$
\left\langle\frac{\partial \mathbf{v}(x, u)}{\partial x}, f(x, u)\right\rangle-\mathbf{v}(x, u)\left\|\frac{\partial \mathbf{v}(x, u)}{\partial u}\right\|_{\star}=\sum_{i=1}^{n} \frac{\partial \mathbf{v}(x, u)}{\partial x_{i}} f_{i}(x, u)-\mathbf{v}(x, u)\left\|\frac{\partial \mathbf{v}(x, u)}{\partial u}\right\|_{\star}=0
$$

on the graph of $U$, where $\|p\|_{\star}:=\sup _{\|v\| \leq 1}\langle p, v\rangle$ denotes the dual norm (in the generalized sense of Barron-Jensen \& Frankowska, [13, Barron \& Jensen], [9, 26, 27, Frankowska]).

To give a meaning to such generalized solutions, we introduce the (contingent) epiderivative $D_{\uparrow} \alpha(x, u)$ of $\alpha$ at $(x, u) \in \operatorname{Dom}(\alpha)$ defined by 


$$
D_{\uparrow} \alpha(x, u)\left(x^{\prime}, u^{\prime}\right):=\liminf _{h \rightarrow 0+, \xi \rightarrow x^{\prime}, \nu \rightarrow u^{\prime}} \frac{\alpha(x+h \xi, u+h \nu)-\alpha(x, u)}{h}
$$

and its convex hull $\widehat{D_{\uparrow}} \alpha(x, u)$, defined as the largest lower semicontinuous function smaller than or equal to $D_{\uparrow} \alpha(x, u)$. We recall that the epigraphs of these epiderivatives are related to the contingent cones to the epigraph of the function by the following formulas:

$$
\mathcal{E} p\left(D_{\uparrow} \alpha(x, u)\right)=T_{\mathcal{E} p(\alpha)}(x, u, \alpha(x, u)) \& \mathcal{E} p\left(\widehat{D_{\uparrow}} \alpha(x, u)\right)=\overline{\operatorname{co}}\left(T_{\mathcal{E} p(\alpha)}(x, u, \alpha(x, u))\right)
$$

Theorem 3.5 Assume that $f$ is continuous, that the graph of $U$ is closed and that the inertia function is continuous on its domain. Then it is the smallest nonnegative lower semicontinuous fonction $\mathbf{v}$ defined on $\operatorname{Graph}(U)$ solution to

$$
\forall(x, u) \in \operatorname{Dom}(\mathbf{v}), \quad \inf _{\left\|u_{1}\right\| \leq \mathbf{v}(x, u)} D_{\uparrow} \mathbf{v}(x, u)\left(f(x, u), u_{1}\right) \leq 0
$$

Proof - This a consequence of Theorem 2.4 when $K:=\operatorname{Graph}(U) \times \mathbb{R}_{+}, C:=\emptyset$ and the control system is metasystem (7).

It states that the epigraph $\mathcal{E} p(\alpha)$ of the inertia function, which is the viability kernel of $\operatorname{Graph}(U) \times \mathbb{R}_{+}$under metasystem (7) thanks to Theorem 3.2, is the largest closed subset $\mathcal{V}:=\mathcal{E} p(\mathbf{v})$ of $\operatorname{Graph}(U) \times \mathbb{R}_{+}$such that for every $(x, u, c) \in \mathcal{V}:=\mathcal{E} p(\mathbf{v})$,

$$
\exists\left\|u_{1}\right\| \leq \mathbf{v}(x, u) \text { such that }\left(f(x, u), u_{1}, 0\right) \in T_{\mathcal{E} p(\mathbf{v})}(x, u, c)
$$

If $c=\mathbf{v}(x, u)$, we deduce from formula

$$
T_{\mathcal{E} p(\mathbf{v})}(x, u, \mathbf{v}(x, u))=\mathcal{E} p\left(D_{\uparrow} \mathbf{v}(x, u)\right)
$$

that

$$
\left\|u_{1}\right\| \leq \mathbf{v}(x, u) \text { and } D_{\uparrow} \mathbf{v}(x, u)\left(f(x, u), u_{1}\right) \leq 0
$$

Since the epiderivative is lower semicontinuous and the balls are compact, this is equivalent to writing that

$$
\forall(x, u) \in \operatorname{Dom}(\mathbf{v}), \quad \inf _{\left\|u_{1}\right\| \leq \mathbf{v}(x, u)} D_{\uparrow} \mathbf{v}(x, u)\left(f(x, u), u_{1}\right) \leq 0
$$

This concludes the proof. 
Definition 3.6 The metaregulation map $G: \mathcal{E} p(\alpha) \leadsto \mathcal{U}$ is the regulation map of metasystem (7): It associates with any $(x, u, c) \in \operatorname{Graph}(U) \times \mathbb{R}_{+}$the subset $G(x, u, c)$ of metacontrols $u_{1}$ such that

$$
\left\|u_{1}\right\| \leq c \text { and }\left(f(x, u), u_{1}, 0\right) \in \overline{\mathrm{co}}\left(T_{\mathcal{E} p(\alpha)}(x, u, c)\right)
$$

Proposition 3.7 Let us assume that the inertia function $\alpha$ is continuous on its domain. Then the metaregulation map can be written in the following form

$$
G(x, u, c)=\left\{\begin{array}{lr}
\operatorname{Dom}\left(D_{\uparrow} \alpha(x, u)\right) \cap c B & \text { if } \alpha(x, u)<c \\
\left\{u_{1} \in \alpha(x, u) B \text { such that } \widehat{D_{\uparrow}} \alpha(x, u)\left(f(x, u), u_{1}\right) \leq 0\right\} & \text { if } \alpha(x, u)=c
\end{array}\right.
$$

Therefore, all evolutions of metasystem (7) starting from $(x, u, c)$ viable in $\operatorname{Graph}(U) \times \mathbb{R}_{+}$ are regulated by the system of differential inclusions

$$
\begin{cases}(i) & x^{\prime}(t)=f(x(t), u(t)) \\ (i i) & u^{\prime}(t) \in G(x(t), u(t))\end{cases}
$$

Proof - The formula follows from the definition of the metaregulation map $G$, the formula

$$
\overline{\mathrm{CO}}\left(T_{\mathcal{E} p(\alpha)}(x, u, c)\right)=\mathcal{E} p\left(\widehat{D_{\uparrow}} \alpha(x, u)\right)
$$

and the above characterization of the contingent cone of the epigraph of $\alpha$ when it is continuous on its domain.

Definition 3.8 Since the values $G(x, u, c)$ of the metaregulation map $G$ are obviously closed and convex, we denote by $g^{0}(x, u, c) \in G(x, u, c)$ the element of minimal norm of $G(x, u, c)$, defining the minimal selection $g^{0}$ of the metaregulation map $G$.

The evolutions governed by system of differential equations

$$
\begin{cases}(i) & x^{\prime}(t)=f(x(t), u(t)) \\ (\text { ii }) & u^{\prime}(t)=g^{0}(x(t), u(t), y(t)) \\ (\text { iii }) & y^{\prime}(t)=0\end{cases}
$$

starting from $(x, u, c)$ and viable in the epigraph of the inertia function are called heavy evolutions with inertia threshold $c$.

Theorem 3.9 If the inertia function $\alpha$ is continuous on its domain and if the function $\gamma$ defined by

$$
\gamma(x, u):=\inf _{u_{1} \in \alpha(x, u) B \text { such that } D_{\uparrow} \alpha(x, u)\left(f(x, u), u_{1}\right) \leq 0}\left\|u_{1}\right\|
$$


is upper semicontinuous, then from any $(x, u, c) \in \mathcal{E} p(\alpha)$ starts a heavy evolution governed by the system of differential equations (8) viable in the epigraph of the inertia function.

Proof - Observe that

$$
\left\|g^{0}(x, u, y)\right\|= \begin{cases}\{0\} & \text { if } \quad \alpha(x, u)<y \\ \gamma(x, u) & \text { if } \quad \alpha(x, u)=y\end{cases}
$$

is upper semicontinuous, so that the auxiliary system

$$
\begin{cases}(i) & x^{\prime}(t)=f(x(t), u(t)) \\ (i i) & u^{\prime}(t) \in\left\|g^{0}(x(t), u(t), y(t))\right\| B \\ (\text { iii }) & y^{\prime}(t)=0\end{cases}
$$

is Marchaud. By construction, the epigraph of the inertia function is viable under this auxiliary system, so that, from any initial $(x, u, c)$ starts an evolution viable in $\mathcal{E} p(\alpha)$ satisfying

$$
\left\|u^{\prime}(t)\right\| \leq\left\|g^{0}(x(t), u(t), c)\right\|
$$

Being viable in $\mathcal{E} p(\alpha)$, it satisfies

$$
u^{\prime}(t) \in G(x(t), u(t), c)
$$

This implies that for almost all $t \geq 0$,

$$
u^{\prime}(t)=g^{0}(x(t), u(t), c)
$$

This concludes the proof.

\subsection{Behavior of Heavy Evolutions}

We now describe the behavior of heavy evolutions for a inertia threshold $c$.

Assume that $\alpha(x, u)<c$. Since $\alpha$ is assumed to be continuous, we deduce that whenever $\alpha(x(t), u(t))<c$, the velocity $u^{\prime}(t)=0$ so that the control remains constant. Hence $x(\cdot)$ is regulated by a constant control as long as $\alpha(x(t), u)<c$. Let $t^{\star}$ be the first instant (kairos) when $\alpha\left(x\left(t^{\star}\right), u\right)=c$. Then we know that $u^{\prime}\left(t^{\star}\right)=g^{0}\left(x\left(t^{\star}\right), u\left(t^{\star}\right), \alpha\left(x\left(t^{\star}\right), u\left(t^{\star}\right)\right)\right)$, so that $\left\|u^{\prime}\left(t^{\star}\right)\right\|=c$.

If the map $f$ is Lipschitz, then the Quincampoix Theorem (see [33, Quincampoix]) implies that $\left.\alpha(x(t), u(t))=\alpha\left(x\left(t^{\star}\right), u\left(t^{\star}\right)\right)\right)=c$ as long as $(x(t), u(t)) \in \operatorname{Int}(\operatorname{Graph}(U))$ since the boundary of the epigraph of $\alpha$, which is equal to the graph of $\alpha$ whenever it is continuous, enjoys the barrier property: The evolution remains in the boundary as long as $(x(t), u(t), c)$ belongs to the interior of $\operatorname{Graph}(U) \times \mathbb{R}_{+}$. It may change only when the state control pair $(x(t), u(t))$ hits the boundary of $\operatorname{Graph}(U)$.

If $c=0$, then the viability niche $\Xi(0, u)$ is viable under differential condition $x^{\prime}(t)=$ $f(x(t), u)$ with constant control. 


\section{Example: Evolution of the Biomass of a Renewable Re- source}

We illustrate some of the basic concepts of viability theory with the study of the evolution of the biomass of one population (of renewable resources, such as fishes in fisheries: see [21, Cury, Mullon \& Shannon] [20, Cury, Mullon, Garcia \& Shannon] for a deeper study of applications of viability theory to fisheries ). It provides simple one-dimensional regulated systems. The mention of biomass is just used to provide some intuition to the mathematical concepts and results, but not the other way around, as a "model" of what happens in this mysterious and difficult field of management of renewable resources. Many other interpretations of the variables and controls presented could have been chosen, naturally. In any case, whatever the chosen interpretation, these one-dimensional systems are far too simplistic for their conclusions to be taken seriously.

\subsection{From Malthus to Verhulst and Beyond}

We assume that there is a constant supply of resources, no predators and limited space: at each instant $t \geq 0$, the biomass $x(t)$ of the population must remain confined in an interval $K:=[a, b]$ where $0<a<b$ describing the environment. The maximal size $b$ that the biomass can achieve is called the carrying capacity in the specialized literature.

We say that an evolution $x(\cdot): t \mapsto x(t)$ is viable in environment $K$ if

$$
\forall t \geq 0, x(t) \in K
$$

The dynamics governing the evolution of the biomass are unknown, really. However, several models have been proposed. They are all particular cases of a general dynamical system of the form

$$
x^{\prime}(t)=\widetilde{u}(x(t)) x(t)
$$

where $\widetilde{u}:[a, b] \mapsto \mathbb{R}$ is a mathematical translation of the growth rate of the biomass of the population feeding back on the biomass (the specialists of these fields prefer to study growth rates than velocities, as in mechanics or physics). Such a map $\widetilde{u}$ is usually called a feedback (also called "retroaction, closed-loop control" in control theory).

The scarcity of resources sets a limit to population growth: This is the simplest example of a viability problem.

We say that the environment $K:=[a, b]$ is viable under differential equation (9) if for every initial state $x \in K$, the evolution $x(\cdot): t \mapsto x(t)$ starting from $x$ is viable in $K$.

The question soon arose to know whether the environment $K:=[a, b]$ is viable under differential equation (9) associated with such or such feedback $\widetilde{u}$ proposed by specialists in population dynamics. 
Another question, which we answer in this paper, is in some sense "inverse": Given an environment, the viability property and maybe other properties required on the evolutions, what are all the feedbacks $\widetilde{u}$ under which these properties are satisfied? Answering the second question automatically answers the first one.

1. Thomas Malthus was the first one to address this viability problem and came up a negative answer. He advocated in 1798 to choose a constant positive growth rate $\widetilde{u}_{0}(x)=r>0$, leading to an exponential evolution $x(t)=x e^{r t}$ starting at $x$. It leaves the interval $[a, b]$ at finite time $t^{\star}:=\frac{1}{r} \log \left(\frac{b}{x}\right)$. In other words, no bounded interval can be viable under Malthusian dynamics. This is the price to pay for linearity of the dynamics of the population:"Population, when unchecked, increases in a geometrical ratio", as he concluded in his celebrated An essay on the principle of population (1798). He thus was worried by the great poverty of his time, so that he finally recommended "moral restraint" to stimulate savings, diminish poverty, maintain wages above the minimum necessary, and catalyze happiness and prosperity.

For overcoming this pessimistic conclusions, other explicit feedbacks have next been offered for providing evolutions growing fast when the population is small and declining when it becomes large to compensate for the never ending expansion of the Malthusian model.

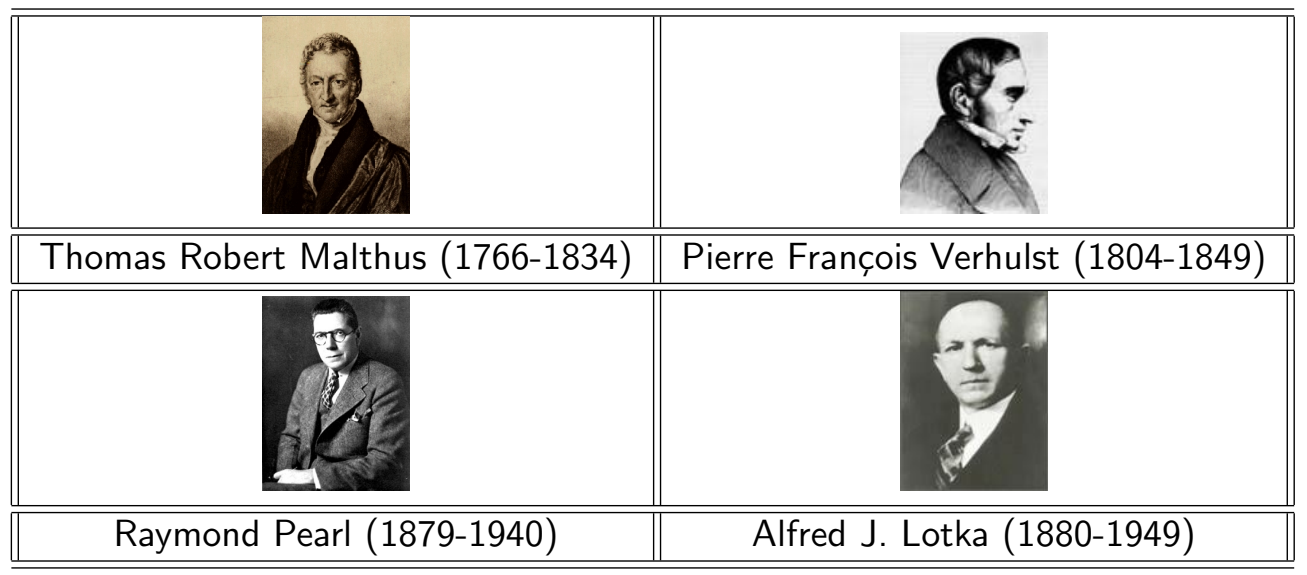

2. The Belgium mathematician Pierre-François Verhulst proposed in 1838 the Verhulst feedback of the form

$$
\widetilde{u}_{1}(x):=r(b-x) \text { where } r>0
$$

after he had read Thomas Malthus' Essay. It was rediscovered in 1920 by Raymond Pearl and again in 1925 by A. J. Lotka who called it the law of population growth. The environment $K$ is viable under the associated purely logistic Verhulst equation $x^{\prime}(t)=$ 
$r x(t)(b-x(t))$. The solution starting from $x \in[a, b]$ is equal to $x(t)=\frac{b x}{x+(b-x) e^{-r t}}$. It is known as the "sigmoid" because of its famous $S$-shape, remains confined in the interval $[a, b]$ and converges to the carrying capacity $b$ when $t \mapsto+\infty$. The logistic model and the $S$-shape graph of its solution became very popular since the 1920's and stood as the evolutionary model of a large manifold of growths, from the tail of mice to the size of men.

3. However, other examples of explicit feedbacks have been used in population dynamics. For instance, the environment $K$ is viable under the following feedbacks: $\widetilde{u}_{2}(x):=$ $e^{r(b-x)}-1$, a continuous analogue of a discrete time model proposed by Ricker and May, $\widetilde{u}_{3}(x):=r(b-x)^{\alpha}$, a continuous analogue of a discrete-time model proposed by Hassel and May, the feedback $\widetilde{u}_{4}(x):=r\left(\frac{1}{\sqrt{x}}-\frac{1}{\sqrt{b}}\right)$, etc. 


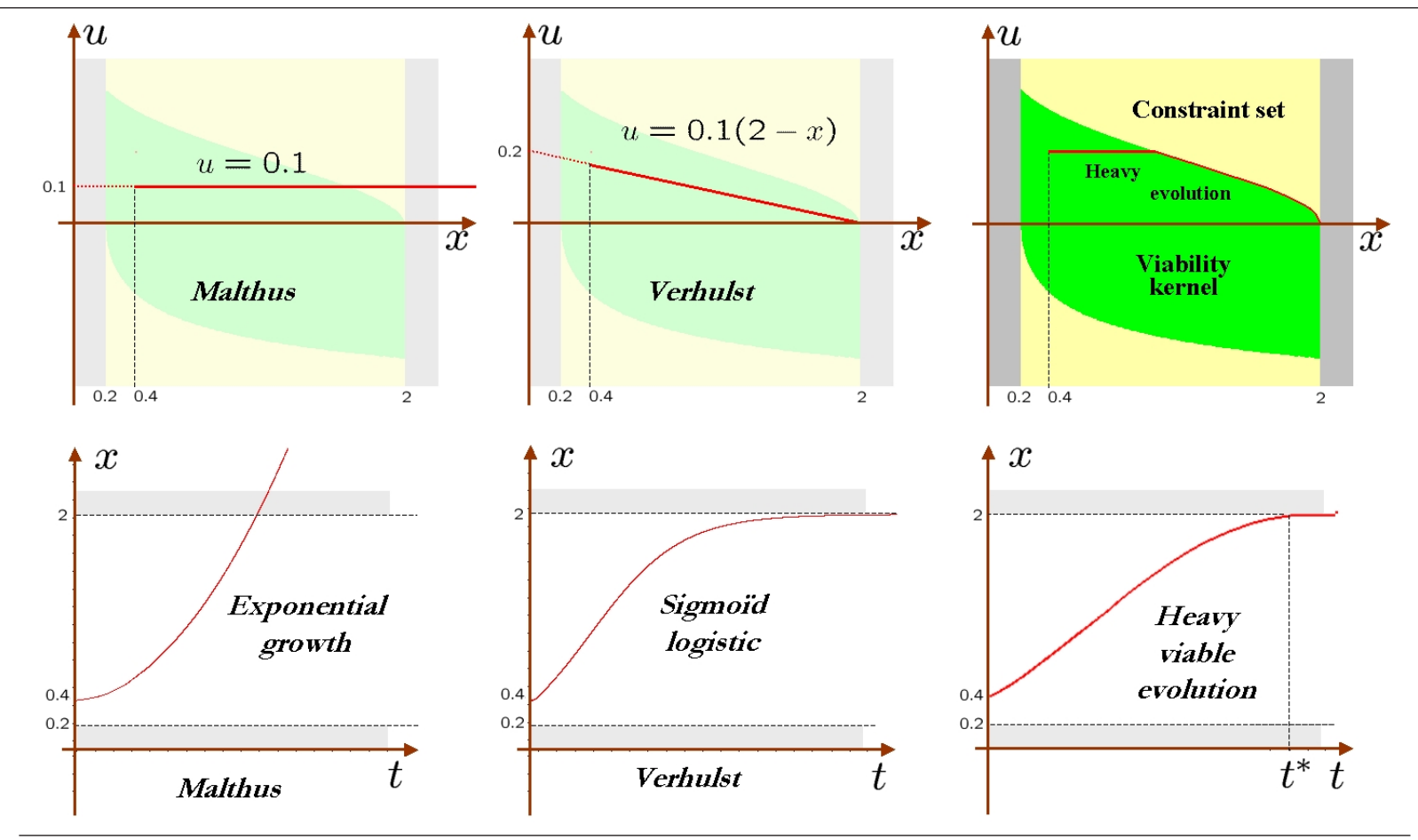

Figure 4.1 The three figures of the top line illustrate the curves representing three examples of feedbacks. The coordinates represent the biomass $x$ and the growth rate $u$ of the population.

The left figures shows the (constant) Malthusian feedback, the center one the affine Verhulst feedback and the right one the heavy feedback. The Malthus and Verhulst feedbacks are given a priori. The Malthus feedback is not viable in the viability interval $[0.2,2]$, but the Verhulst feedback is. The heavy feedback is computed a posteriori from viability, flexibility and inertia requirements. For that purpose, we have to compute the viability kernel obtained with the Viability Kernel Algorithm.

The three figures of the bottom line provide the corresponding evolutions. The coordinates represent the time $t$ and the biomass $x$. The evolution is exponential ii the Malthusian case, and thus, leaves the viability interval, the logistic sigmoid in the Verhulst case, which converges asymptotically to the upper bound of the viability interval. It is reached in finite time $t^{*}$ by an heavy evolution, starting as an exponential, and next slowed down to reach the upper bound with a growth rate equal to zero (an equilibrium).

Instead of finding one feedback $\widetilde{u}$ satisfying the above viability requirements by trial and error, we proceed systematically for designing feedbacks by leaving the choice of the growth rates open, regarding them as controls (regulation parameters) of the regulated system

$$
x^{\prime}(t)=u(t) x(t)
$$

where the control $u(t)$ is chosen at each time $t$ for governing evolutions confined in the interval $[a, b]$.

We denote by $\mathcal{P}(x, u)$ the set of solutions to system viable in the interval $[a, b]$. The 
inertia function is defined by

$$
\alpha(x, u):=\inf _{x(\cdot) \in \mathcal{P}(x, u)} \sup _{t \geq 0}\left|u^{\prime}(t)\right|
$$

The domain $\operatorname{Dom}(\alpha)$ of the inertia function of system $x^{\prime}(t)=u(t) x(t)$ confronted to environment $K:=[a, b]$ is equal to

$$
\operatorname{Dom}(\alpha):=\left(\{a\} \times \mathbb{R}_{+}\right) \cup(] a, b[\times \mathbb{R}) \cup\left(\{b\} \times \mathbb{R}_{-}\right)
$$

and the inertia function is equal to:

$$
\alpha(x, u):=\left\{\begin{array}{lll}
\frac{u^{2}}{2 \log \left(\frac{b}{x}\right)} & \text { if } \quad a \leq x<b \& u \geq 0 \\
\frac{u^{2}}{2 \log \left(\frac{x}{a}\right)} & \text { if } \quad a<x \leq b \& u \leq 0
\end{array}\right.
$$

The epigraph $\mathcal{E} p(\alpha)$ of the inertia function is closed. However, its domain is neither closed nor open (and not even locally compact). The restriction of the inertia function to its domain is continuous.

Remark: - The inertia function is the unique lower semicontinuous solution (in the generalized sense of Barron-Jensen \& Frankowska) to the Hamilton-Jacobi partial differential equation

$$
\left\{\begin{array}{l}
\frac{\partial \alpha(x, u)}{\partial x} u x-\alpha(x, u) \frac{\partial \alpha(x, u)}{\partial u}=0 \text { if } \quad a \leq x<b \& u \geq 0 \\
\frac{\partial \alpha(x, u)}{\partial x} u x+\alpha(x, u) \frac{\partial \alpha(x, u)}{\partial u}=0 \text { if } a<x \leq b \& u \leq 0
\end{array}\right.
$$

on $\operatorname{Dom}(\alpha)$ with discontinuous coefficients. Indeed, the partial derivatives of these two inertia functions are equal to

$$
\frac{\partial \alpha(x, u)}{\partial x}:=\left\{\begin{array}{ll}
\frac{u^{2}}{2 x\left(\log \left(\frac{b}{x}\right)^{2}\right)} & \text { if } u \geq 0 \\
-\frac{u^{2}}{2 x\left(\log \left(\frac{x}{a}\right)^{2}\right)} & \text { if } \quad u \leq 0
\end{array} \quad \& \frac{\partial \alpha(x, u)}{\partial u}:= \begin{cases}\frac{u}{\log \left(\frac{b}{x}\right)} & \text { if } \quad u \geq 0 \\
\frac{u}{\log \left(\frac{x}{a}\right)} & \text { if } \quad u \leq 0\end{cases}\right.
$$

Observe that $\frac{\partial \alpha(x, u)}{\partial u}$ is positive when $u>0$ and negative when $u<0$.

We deduce from the above lemma the analytical formulas of the inert regulation and crisis maps:

Proposition 4.2 For system $x^{\prime}(t)=u(t) x(t)$, the inert regulation map

$$
(c, x) \leadsto R(c ; x):=\{u \in \mathbb{R} \text { such that } \alpha(x, u) \leq c\}
$$



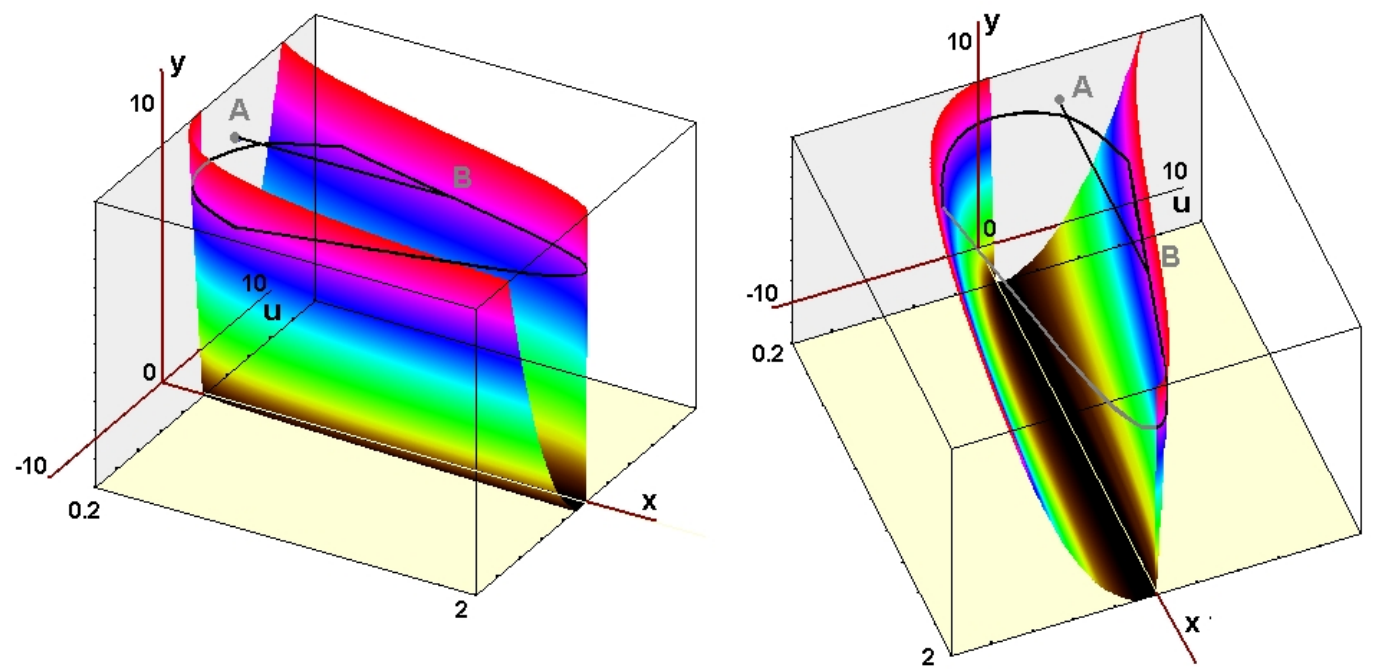

Figure 2: Inertia Function

associated with the inertia function is equal to

$$
R(c, x):= \begin{cases}{\left[0, \sqrt{2 c \log \left(\frac{b}{a}\right)}\right]} & \text { if } \quad x=a \\ {\left[-\sqrt{2 c \log \left(\frac{x}{a}\right)}, \sqrt{2 c \log \left(\frac{b}{x}\right)}\right]} & \text { if } \quad a<x<b \\ {\left[-\sqrt{2 c \log \left(\frac{b}{a}\right)}, 0\right]} & \text { if } \quad x=b\end{cases}
$$

The crisis map $(c, u) \leadsto \Xi(c ; u):=\{x \in[a, b]$ such that $\alpha(x, u)=c\}$ is equal to

$$
\Xi(c, u):=\left\{\begin{array}{lll}
b e^{\frac{u^{2}}{2 c}} & \text { if } u>0 \\
a e^{\frac{u^{2}}{2 c}} & \text { if } u<0
\end{array}\right.
$$

if $c>0$ and to

$$
\Xi(0, u):=\left\{\begin{array}{lll}
{[a, b]} & \text { if } & u=0 \\
\emptyset & \text { if } & u \neq 0
\end{array}\right.
$$

if $c=0$

Since the epigraph of the inertia function is the viability kernel of the "metaenvironment" 
$\mathcal{K}:=[a, b] \times \mathbb{R}_{+} \times \mathbb{R}_{+}$under the metasystem

$$
\begin{cases}(i) & x^{\prime}(t)=u(t) x(t) \\ (i i) & u^{\prime}(t)=v(t) \\ (i i) & y^{\prime}(t)=0 \\ & \text { where }|v(t)| \leq y(t)\end{cases}
$$

the Viability Theorem provides the analytical formula of the metaregulation map $(x, u, c) \leadsto$ $G(x, u, c)$ associating with any metastate $(x, u, c)$ the set $G(x, u, c)$ of metacontrols governing the evolution of evolutions with finite inertia:

1. Case when $\alpha(x, u)<c$. Then

$$
G(x, u, c):=\left\{\begin{array}{lll}
{[0, c]} & \text { if } \quad x=a \\
{[-c,+c]} & \text { if } \quad a<x<b \\
{[-c, 0]} & \text { if } \quad x=b
\end{array}\right.
$$

2. Case when $\alpha(x, u)=c$. Then

$$
G(x, u, c):= \begin{cases}-\alpha(x, u) & \text { if } \quad u \geq 0 \& a \leq x<b \\ \alpha(x, u) & \text { if } u \geq 0 \& a<x \leq b\end{cases}
$$

The minimal selection $g^{\circ}(x, u, c) \in G(x, u, c)$ is equal to $g^{\circ}(x, u, c)=0$ if $\alpha(x, u)<c$ and to

$$
g^{\circ}(x, u, \alpha(x, u)):= \begin{cases}-\alpha(x, u) & \text { if } \quad u \geq 0 \& a \leq x<b \\ \alpha(x, u) & \text { if } \quad u \geq 0 \& a<x \leq b\end{cases}
$$

if $\alpha(x, u)=c$, i.e., if $x \in \Xi(c, u)$ is located in the crisis zone of the control $u$ at inertia threshold $c$.

Although the minimal selection $g^{\circ}$ is not continuous, for any initial pair $(x, u) \in \operatorname{Dom}(\alpha)$ in the domain of the inertia function, system of differential equations

$$
\begin{cases}(i) & x^{\prime}(t)=u(t) x(t) \\ (i i) \quad u^{\prime}(t)=g^{\circ}(x(t), u(t), c)\end{cases}
$$

has solutions which are called heavy viable evolutions of initial system 10 . The trajectory of this heavy evolution is shown on the graph of the inertia function displayed in Figure 2.

\section{Management of Renewable Resources}

Let us consider a given non negative growth rate feedback $\widetilde{u}$ governing the evolution of the biomass of a renewable resource $x(t) \geq a>0$, through differential equation : $x^{\prime}(t)=$ 


\section{Figure 3:}

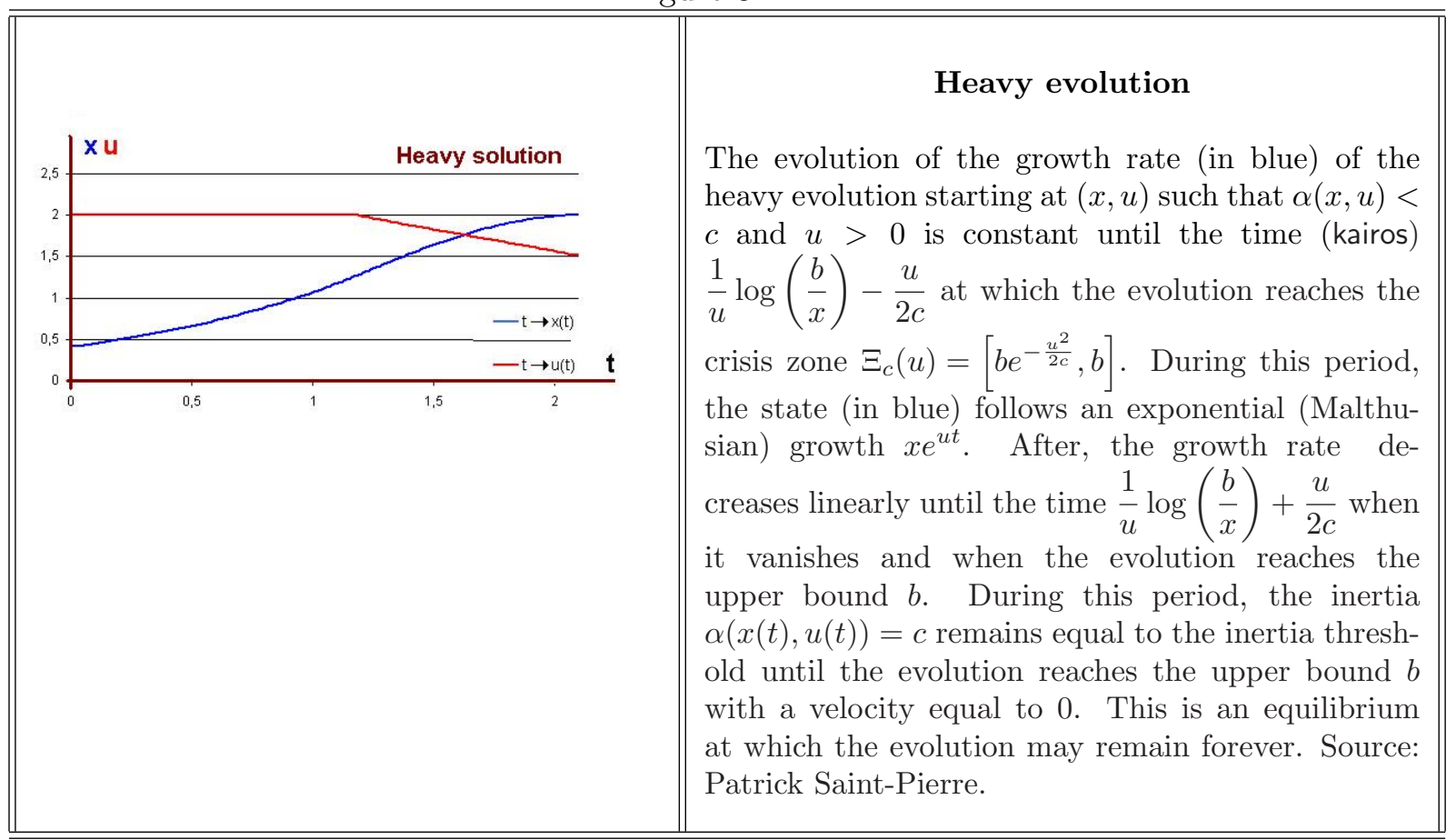


$\widetilde{u}(x(t)) x(t)$. We shall take as examples the Malthusian feedback $u_{0}(x):=u$, the Verhulst feedback $u_{1}(x):=r(x-b)$ and the inert feedback $\widetilde{u}_{5}(x):=r \sqrt{2 \log \left(\frac{b}{x}\right)}$.

The evolution is slowed down by industrial activity which depletes it, such as fisheries.

We denote by $v \in \mathbf{R}_{+}$the industrial effort for exploiting the renewable resource, playing now the role of the control. Naturally, the industrial effort is subjected to state-dependent constraints $V(x)$ describing economic constraints.

We integrate the ecological constraint by setting $V(x)=\emptyset$ whenever $x<a$.

Hence the evolution of the biomass is regulated by the control system

$$
\begin{cases}(i) & x^{\prime}(t)=x(t)(\widetilde{u}(x(t))-v(t)) \\ (i i) & v(t) \in V(x(t))\end{cases}
$$

We denote by $\mathcal{V}_{\widetilde{u}}(x, v)$ the set of solutions to system (13). The inertia function is defined by

$$
\beta_{\widetilde{u}}(x, v):=\inf _{x(\cdot) \in \mathcal{V}_{\widetilde{u}}(x, v)} \sup _{t \geq 0}\left|u^{\prime}(t)\right|
$$

This function is characterized as the viability kernel of the "metaenvironment" $\mathcal{K}:=$ $[a, b] \times \mathbb{R}_{+} \times \mathbb{R}_{+}$under the metasystem

$$
\begin{cases}(i) & x^{\prime}(t)=(\widetilde{u}(x(t))-v(t)) x(t) \\ (i i) & v^{\prime}(t)=w(t) \\ (i i) & y^{\prime}(t)=0 \\ & \text { where }|w(t)| \leq y(t)\end{cases}
$$

We observe that the inertia function vanishes on the equation line:

$$
\beta_{\widetilde{u}}(x, \widetilde{u}(x))=0
$$

It is identically equal to 0 if for any $x \geq a, \widetilde{u}(x) \geq \mathbf{v}(x)$ and identically infinite if for any $x \geq a, \widetilde{u}(x)<\mathbf{v}(x)$.

The basic economic model was originated by Graham and taken up by Schaeffer. They assumed that the exploitation rate is proportional to the biomass and the economic activity: viability constraints are described by economic constraints

$$
\forall t \geq 0, c v(t)+C \leq \gamma v(t) x(t)
$$

where $C \geq 0$ is a fixed cost, $c \geq 0$ the unit cost of economic activity and $\gamma \geq 0$ the price of the resource. We also assume that

$$
\forall t \geq 0, \quad 0 \leq v(t) \leq \bar{v}
$$


where $\bar{v}>\frac{C}{\gamma x-c}$ is the maximal exploitation effort. Hence the Graham-Schaeffer constraints are summarized under the set-valued map $V:\left[a, \infty\left[\sim \mathbb{R}_{+}\right.\right.$defined by

$$
\forall x \geq a, V(x):=\left[\frac{C}{\gamma x-c}, \bar{v}\right]
$$

More generally, we assume that there exists a decreasing positive map $\mathbf{v}:[a, b] \mapsto[0, \bar{v}]$ such that

$$
\forall x \in[a, \infty[, V(x):=[\mathbf{v}(x), \bar{v}]
$$

In any case, the epigraph of the inertia function being a viability kernel, it can be computed the Saint-Pierre Viability Kernel Algorithm. Figure 4 provides the level sets of the inertia function for the the Verhulst and inert feedbacks respectively.
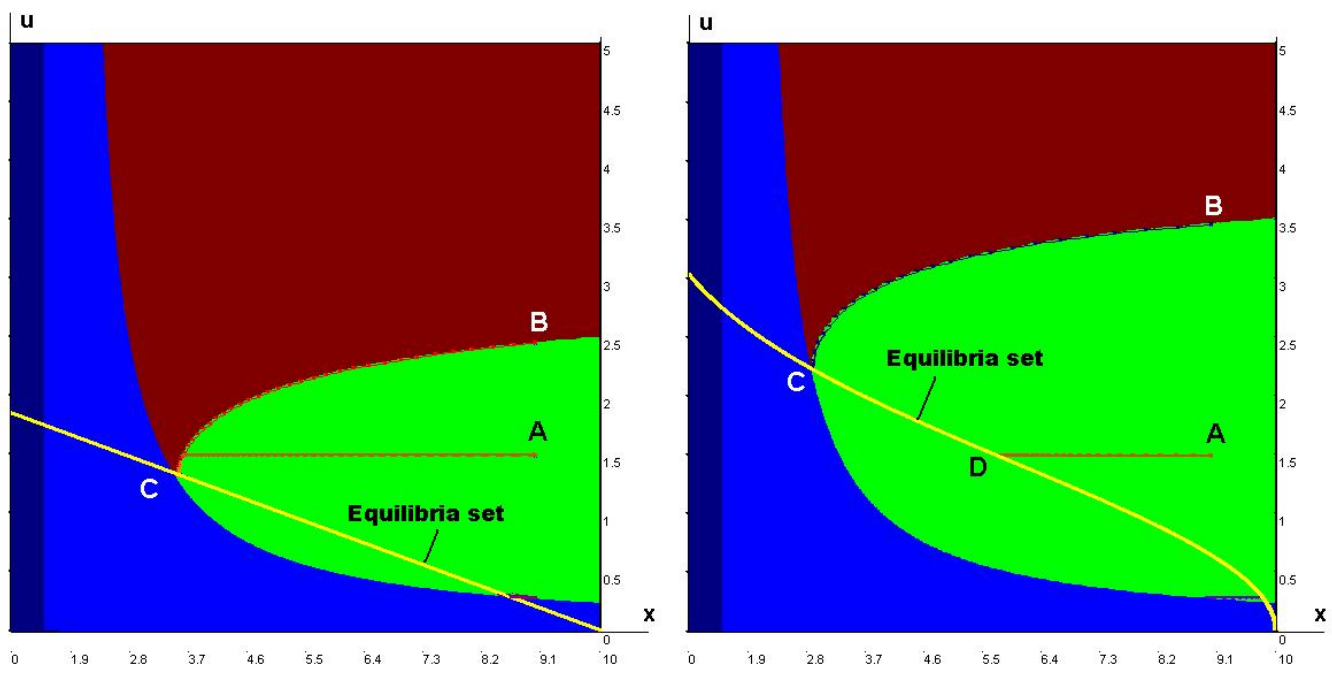

Figure 4: Regulation Maps and heavy solutions under Verhulst-Schaeffer and Inert-Schaeffer Metasystems $x^{\prime}(t)=r x(t)\left(1-\frac{x(t)}{b}\right)-v(t) x(t)$ and $x^{\prime}(t)=$ $x(t)\left(r \sqrt{2 \log \left(\frac{b}{x(t)}\right)}-v(t)\right)$ respectively. The equilibrium lines are the graphs of $r\left(1-\frac{x}{b}\right)$ and $r \sqrt{2 \log \left(\frac{b}{x}\right)}$. Heavy evolutions stop when their trajectories hit the equilibrium line. 
Using the Malthusian (constant) feedbacks $\widetilde{u}_{0}(x) \equiv u$ for the growth of the renewable resource allows us to provide analytical formula of the inertia function for any decreasing exploitation function $\mathbf{v}(x)$ such as the Graham-Schaeffer one. Let us define by $\nu(u)$ the root of the equation $\mathbf{v}(x)=u$ and set $a(u):=\max (a, \nu(u))$.

The inertia function is equal to:

$$
\beta_{u}(x, v):= \begin{cases}\frac{(v-u)^{2}}{2 \log \left(\frac{c}{a(u)}\right)} & \text { if } \quad v \geq u \text { and } x \geq a(u) \\ 0 & \text { if } \quad \mathbf{v}(x) \leq v \leq u \text { and } x \geq a(u)\end{cases}
$$

The epigraph $\mathcal{E} p\left(\beta_{u}\right)$ of the inertia function is closed. However, its domain is neither closed nor open (and not even locally compact). The restriction of the inertia function to its domain is continuous.

Remark: - The inertia function is a solution to the Hamilton-Jacobi partial differential equation when $v \geq u$ :

$$
\frac{\partial \beta_{u}(x, v)}{\partial x}(u-v) x-\beta_{u}(x, v) \frac{\partial \beta_{u}(x, v)}{\partial v}=0
$$

Indeed, the partial derivatives of these two inertia functions are equal to

$$
\frac{\partial \beta_{u}(x, v)}{\partial x}:=-\frac{(v-u)^{2}}{2 x\left(\log \left(\frac{x}{a(u)}\right)^{2}\right)} \& \frac{\partial \beta_{u}(x, v)}{\partial v}:=\frac{v-u}{\log \left(\frac{x}{a(u)}\right)}
$$

Observe that $\frac{\partial \beta_{u}(x, v)}{\partial v}$ is positive when $v>u$ and negative when $v<u$.

Proposition 5.1 For system $x^{\prime}(t)=(u-v(t)) x(t)$, the inert regulation map

$$
(c, x) \leadsto R(c ; x):=\left\{v \in \mathbb{R} \text { such that } \beta_{u}(x, v) \leq c\right\}
$$

associated with the inertia function is equal to

$$
R(c, x):=\left[\mathbf{v}(x), u+\sqrt{2 c \log \left(\frac{b x}{a(u)}\right)}\right] \text { if } a(u) \leq x
$$

The crisis map $(c, u) \leadsto \Xi(c ; v):=\left\{x \in[a, b]\right.$ such that $\left.\beta_{u}(x, v)=c\right\}$ is equal to

$$
\Xi((c, v))=[a(u), \xi(c, v)] \text { where } \xi(c, v):=a(u) e^{\frac{(v-u)^{2}}{2 c}}
$$

if $c>0$ and to

$$
\Xi(0, v):= \begin{cases}{[a(u),+\infty[} & \text { if } \quad \mathbf{v}(x) \leq v \leq u \\ \emptyset & \text { if } v>u\end{cases}
$$

if $c=0$ 
The Viability Theorem provides the analytical formula of the metaregulation map $(x, v, c) \leadsto$ $G(x, v, c)$ associating with any metastate $(x, v, c)$ the set $G(x, v, c)$ of metacontrols govening the evolution of evolutions with finite inertia:

1. Case when $\beta_{u}(x, v)<c$. Then

$$
G(x, v, c):=\left[-\beta_{u}(x, v),+\beta_{u}(x, v)\right]
$$

2. Case when $\beta_{u}(x, v)=c$ and $v>u$. Then

$$
G(x, v, c):=-\beta_{u}(x, v)
$$

The minimal selection $g^{0}(x, v, c)$ is defined by $g^{0}(x, v, c)=0$ if $\mathbf{v}(x) \leq v<u+\sqrt{2 c \log \left(\frac{b x}{a(u)}\right)}$ and by $g^{0}(x, v, c)=-\beta_{u}(x, v)$ whenever $v=u+\sqrt{2 c \log \left(\frac{b x}{a(u)}\right)}$.

\subsection{Inert Evolutions}

An evolution $(x(\cdot), u(\cdot))$ is said to be inert on a time interval $\left[t_{0}, t_{1}\right]$ if it is regulated by an affine open-loop controls of the form $v(t):=v+w t$, the velocities $v^{\prime}(t)=w$ of which are constant.

The inertia function remains constant over an inert evolution as long as the evolution is viable: On an adequate interval, we obtain

$$
\forall t \in[0, \bar{t}], \beta_{u}\left(\bar{x}(t), \bar{v}_{u}(t)\right)=\beta_{u}(x, v)=c
$$

Let us consider the case when $v>u$.

The velocity governing the inert evolution is constant and equal to $\bar{v}_{u}^{\prime}(t)=-\beta_{u}(x, v)$, so that

$$
\bar{v}_{u}(t)=v \frac{(v-u)^{2} t}{2 \log \left(\frac{x}{a(u)}\right)}
$$

and the evolution of the inert state by

$$
\bar{x}_{u}(t)=x e^{-(v-u) t-\frac{(v-u)^{2} t^{2}}{4 \log \left(\frac{x}{a(u)}\right)}}
$$

The state decreases until it reaches the lower bound $a(u)$ at time

$$
\tau(x, v)=2 \frac{\log \left(\frac{x}{a(u)}\right)}{v-u}
$$


and decreases until it reaches $a(u)$ in finite time.

This inert evolution is governed by the inert feedback

$$
\widetilde{v}(x):=u+\sqrt{2 \log \left(\frac{x}{a(u)}\right)}
$$

\subsection{Heavy Evolutions}

Heavy evolutions $x_{c}(\cdot)$ are obtained when the absolute value $|w(t)|:=\left|v^{\prime}(t)\right|$ of the velocity $w(t):=v^{\prime}(t)$ of the control is minimized at each instant. In particular, whenever the velocity of the control is equal to 0 , the control is kept constant, and if not, it changes as slowly as possible.

The "heaviest" evolutions are thus obtained by constant controls. This is not always possible, because, by taking $v>u$ for instance, the solution $x(t)=x e^{-(v-u) t}$ is viable for $t \leq \frac{\log \left(\frac{x}{a(u)}\right)}{v-u}$. At that time, the control should be changed immediately (with infinite velocity) to any control $v \leq u$. This brutal and drastic measure - which is found in many natural systems - is translated in mathematics by impulse control.

In order to avoid such abrupt changes of controls, we add the requirement that the velocity of the controls is bounded by a velocity bound $c>\beta_{u}(x, v)$.

Starting from $(x, v)$, the state $x_{c}(\cdot)$ of an heavy evolution evolves according

$$
x_{c}(t)=x e^{-(v-u) t}
$$

and reaches $a$ at time $\frac{\log \left(\frac{x}{a(u)}\right)}{v-u}$.

The inertia function $\beta_{u}$ provides the velocity of the controls and increases over the heavy evolution according to

$$
\forall t \in\left[0, \frac{\log \left(\frac{x}{a(u)}\right)}{v-u}\right], \beta_{u}\left(x_{c}(t), v\right)=\frac{(v-u)^{2}}{2\left(\log \left(\frac{x}{a(u))}\right)-(v-u) t\right)}
$$

The derivatives of the inertia function over the inert evolutions are equal to

$$
\frac{d \beta_{u}\left(x_{c}(t), v\right)}{d t}=\frac{(v-u)^{3}}{2\left(\log \left(\frac{x}{a(u)}\right)-(v-u) t\right)^{2}}
$$


The inertia function reaches the given velocity limit $c>\beta_{u}(x, v)$ at

$$
\left\{\begin{array}{l}
\text { warning state } \xi(c, u)=a(u) e^{\frac{(u-v)^{2}}{2 c}} \\
\text { warning time } \sigma_{c}(x, v):=\frac{\log \left(\frac{x}{a(u)}\right)}{v-u}-\frac{v-u}{2 c}
\end{array}\right.
$$

Hence, once a velocity limit $c$ is fixed, the heavy solution evolves with constant control $u$ until the last instant $\sigma_{c}(x, v)$ when the state reaches $\xi_{c}(v)$ and the velocity of the control $\beta_{u}\left(\xi_{c}(v), v\right)=c$. This is the last time when the control remains constant and has to changed by taking

$$
v_{c}(t)=v-c\left(t-\frac{\log \left(\frac{x}{a(u)}\right)}{v-u}+\frac{v-u}{2 c}\right)
$$

Then the evolution $\left(x_{c}(\cdot), v_{c}(\cdot)\right)$ follows the inert solution starting at $\left(\xi_{c}(v),\right)$. It reaches equilibrium $(a(u), u)$ at time

$$
t^{\star}:=\frac{\log \left(\frac{x}{a(u)}\right)}{v-u}+\frac{v-u}{2 c}
$$

Taking $x(t) \equiv a(u)$ and $v(t) \equiv u$ when $t \geq t^{\star}$, the solution may remain at $a(u)$ forever.

For a given inertia bound $c>\beta_{u}(x, v)$, the heavy evolution $\left(x_{c}(\cdot), v_{c}(\cdot)\right)$ is associated with the heavy feedback $\widetilde{v_{c}}$ defined by

$$
\widetilde{v}_{c}(x):= \begin{cases}v & \text { if } \xi(c, u) \leq x \\ u+\sqrt{2 c \log \left(\frac{x}{a(u)}\right)} & \text { if } \quad a(u) \leq x \leq \xi(c, u)\end{cases}
$$

\subsection{Towards Dynamical Games}

Actually, since we do not really know what are the dynamical equations governing the evolution of the resource, this suggest to leave open the choice of the growth rate of the resource and to regard it as a tyche(meaning "chance" in classical Greek, from the Goddess Tyche), or perturbations, disturbances, etc. They could be called "random variables" if this vocabulary were not already confiscated by probabilists. This is why we borrow the term of tychastic evolution to Charles Peirce:

\section{Charles (Sanders) Peirce (1839-1914)}

Charles Peirce introduced the concept of tychastic evolution in a paper published in 1893 under the title evolutionary love.

"Three modes of evolution have thus been brought before us: evolution by fortuitous variation, evolution by mechanical necessity, and evolution by creative love. We may term them tychastic evolution, or tychasm, anancastic evolution, or anancasm, and agapastic evolution, or agapasm. 
Therefore, we just assume that the evolution of the resource is governed by the tychastic control system

$$
x^{\prime}(t)=(u(t)-v(t)) x(t) \text { where } u(t) \in U(x(t), v(t) \in V(x(t))
$$

Let us denote by $\mathcal{P}(x, u, v)$ the set of evolutions governed by the above system. We also introduce a lower semicontinuous $(u, v) \mapsto \mathbf{n}(u, v)$ on the space $\mathcal{U} \times \mathcal{V}$, such as $\mathbf{n}(u, v):=$ $\|u\|+\|v\|$.

Definition 5.2 The guaranteed inertia function $\gamma(x, u, v)$ is defined by

$$
\gamma(x, u, v):=\inf _{\widetilde{v} \in \widetilde{\mathcal{V}} u((\cdot))} \sup _{t \geq 0} \mathbf{n}\left(u^{\prime}(t), v^{\prime}(t)\right)
$$

One can prove that the epigraph of the guaranteed inertia function is the guaranteed viability kernel of an auxiliary tychastic control system we shall define. We refer to papers by Pierre Cardaliaguet, Marc Quincampoix and Patrick Saint-Pierre who studied these questions (see [14, 17, Cardaliaguet], [30, 31, Quincampoix], [18, 19, Cardaliaguet, Quincampoix \& Saint-Pierre]).

\section{Tychastic Systems}

We denote by $X$ and $\mathcal{V}$ two finite dimensional vector spaces, we introduce a single-valued map $f: X \times \mathcal{V} \leadsto X$ and a set-valued map $V: X \leadsto \mathcal{V}$, with which we define system

$$
\begin{cases}(i) & x^{\prime}(t)=f(x(t), v(t)) \\ (i i) & v(t) \in V(x(t))\end{cases}
$$

We denote by $\mathcal{S}(x)$ the subset of evolutions starting at $x$ governed by system 15 and call the set-valued map $\mathcal{S}: x \in X \leadsto \mathcal{S}(x) \subset \mathcal{C}(0, \infty ; X)$ its associated evolutionary system.

Definition 6.1 Let $K \subset X$ be a constrained subset, regarded as an environment and $C \subset K$ be a subset of the environment, regarded as a target.

1. The subset $\operatorname{Inv}_{\mathcal{S}}(K, C)$ of initial states $x_{0} \in K$ such that all evolutions $x(\cdot) \in \mathcal{S}\left(x_{0}\right)$ starting at $x_{0}$ are viable in $K$ for all $t \geq 0$ or viable in $K$ until they reach $C$ in finite time is called the invariance kernel of $K$ with target $C$ under $\mathcal{S}$.

When the target $C=\emptyset$ is the empty set, we say that $\operatorname{Inv}_{\mathcal{S}}(K):=\operatorname{Inv}_{\mathcal{S}}(K, \emptyset)$ is the invariance kernel of $K$. 
2. The subset $\operatorname{Abs}_{\mathcal{S}}(K, C)$ of initial states $x_{0} \in K$ such that all evolutions $x(\cdot) \in \mathcal{S}\left(x_{0}\right)$ starting at $x_{0}$ are viable in $K$ until they reach $C$ in finite time is called the absorption basin of $K$ with target $C$ under $\mathcal{S}$.

When $K=X$ is the whole space, we say that $\operatorname{Abs}_{\mathcal{S}}(X, C)$ is the absorption basin of $C$.

We say that

1. a subset $K$ is invariant under $\mathcal{S}$ if $K=\operatorname{Inv}(K)$,

2. $K$ is invariant outside a target $C \subset K$ under the evolutionary system $\mathcal{S}$ if $K=\operatorname{Inv}(K, C)$ and that $K$ is invariant under $\mathcal{S}$ if $K=\operatorname{Inv}(K)$,

3. $C$ is separated in $K$ if $C=\operatorname{Inv}(K, C)$.

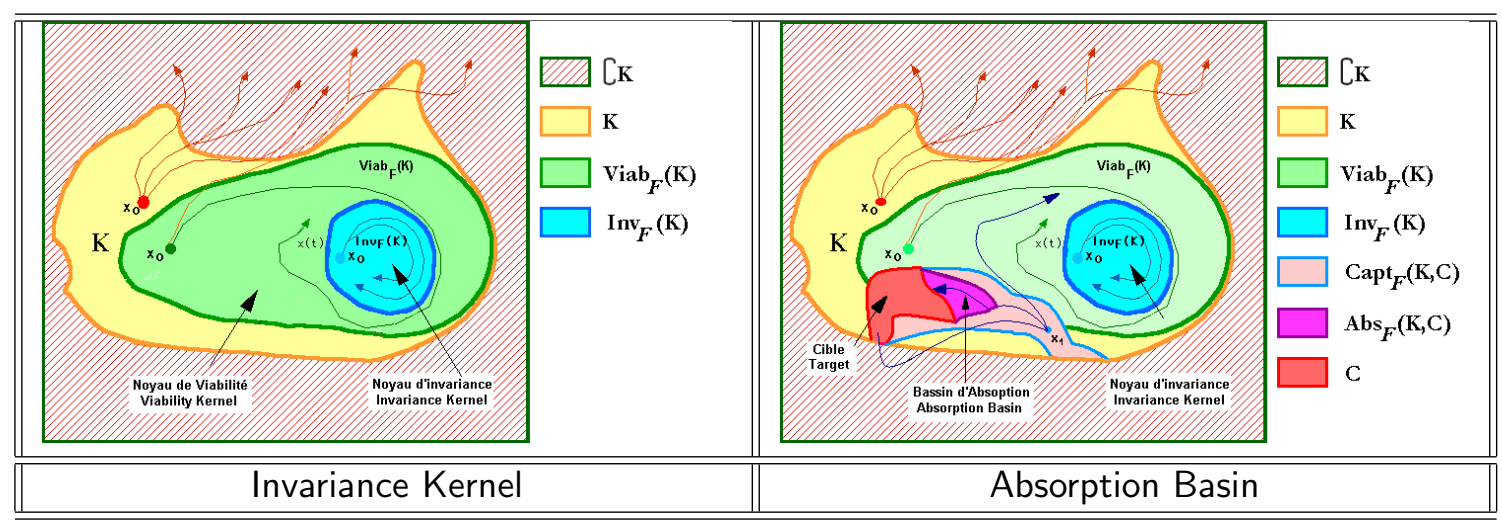

Figure 5: Invariance Kernels and Absorption Basins. The environment (constrained set) is yellow, the target red. The invariance kernel in light blue and the absorption basin in dark pink.

When asking that such a viability/capturability property is satisfied for all evolutions parameterized by evolutions $v(\cdot)$, one cannot regard the parameters $v \in \mathcal{V}$ as controls regulating the evolution of the state $x(\cdot)$ of the system to be viable in the environment forever or until it captures the target in finite time. Such parameters rather play the role of "something random", as in probability theory, to convey an idea sometimes translated by the oxymoron "deterministic uncertainty'. They are often called in "robust control theory" perturbations or disturbances. Such systems provide also mathematical metaphors in other domains (economics, social sciences, biology), as a reasonable alternative to the use of stochastic differential equations for translating mathematically the concept of chance. The availability of the important body of knowledge provided by the theory of stochastic differential equations justified the adoption of the the stochastic approach, despite the fact that in these fields, as in some 
domains of control theory, the uncertainty does not always comply to statistical regularity. Only the knowledge of some bounds or constraints described by the relation $v(t) \in V(x(t))$ is available at each state $x$.

The tychastic map (that could be a fuzzy subset, as it is advocated in [10, Aubin \& Dordan]) provides an alternative mathematical translation of evolution under uncertainty parallel to the usual mathematical translation by a diffusion in the framework of stochastic differential equations.

It easy to check that whenever the complement $K \backslash C:=K \cap \complement C$ of the target $C$ in the environment is a repeller, the invariance kernel with target $C$ and the absorption basin of target $C$ coincide.

We deduce from [9, Aubin \& Catté] the following characterization of invariance kernels:

Theorem 6.2 The invariance kernel $\operatorname{Inv}_{\mathcal{S}}(K, C)$ of $K$ outside the target $C$ is the unique subset between $C$ and $K$ that is both

1. invariant outside $C$ (and is the largest subset $D \subset K$ invariant outside $C$ ),

2. separated in $K$ (and is the smallest subset $D \supset C$ separated in $K$ ):

$$
\operatorname{Inv}_{\mathcal{S}}\left(K, \operatorname{Inv}_{\mathcal{S}}(K, C)\right)=\operatorname{Inv}_{\mathcal{S}}(K, C)=\operatorname{Inv}_{\mathcal{S}}\left(\operatorname{Inv}_{\mathcal{S}}(K, C), C\right)
$$

and thus, the unique bilateral fixed point $D$ between $C$ and $K$ in the sense that

$$
D=\operatorname{Inv}_{\mathcal{S}}(K, D)=\operatorname{Inv}_{\mathcal{S}}(D, C)
$$

The same properties are shared by the absorption basins.

The Invariance Theorem characterizes absorption basins through tangential conditions involving tangent cones (contingent cone) to a subset $K$ at a point $x \in K$.

Theorem 6.3 Assume that the dynamics $f$ and the tychastic map $V$ are Lipschitz and that the target $C$ and the environment $K$ are closed. Then the invariance kernel $\operatorname{Inv}_{\mathcal{S}}(K, C)$ is the largest closed subset $D$ between $C$ and $K$ satisfying the tangential condition

$$
\forall x \in D \backslash C, \forall v \in V(x), \quad f(x, v) \in T_{D}(x)
$$

Furthermore, the Viability Theorem implies that if the assumptions of Theorem 2.4 are satisfied, if the tychastic system is Marchaud, if $K \backslash C$ is a repeller and that if the interior of the target is closed, that the invariance $\operatorname{kernel}^{\operatorname{Inv}_{\mathcal{S}}}(K, C)$ is the unique subset $D$ between $C$ and $K$ satisfying tangential condition 17 and tangential condition

$$
\forall x \in \operatorname{Int}(K) \backslash \operatorname{Int}(D), \exists v \in V(x) \text { such that } f(x, v) \in T_{\overline{\mathrm{C} D}}(x)
$$




\section{Tychastic and Stochastic Invariance}

The reason to use this terminology is to underline the comparison with stochastic differential equations. Concepts of invariance kernels and absorption basins can also be defined for usual stochastic differential equations. : Stochastic invariance kernels and absorption basins are particular cases of invariance kernels and absorption basins under systems of the form 15.

To be precise, let us consider random events $\omega \in \Omega$, where $(\Omega, \mathcal{F}, \mathbb{P})$ is a probability space, instead of tyches $v(\cdot) \in V(x(\cdot))$.

Denote by $\mathbb{X}_{\omega}^{x}(t):=\mathbb{X}(x, \omega)(t)$ the solution starting at $x$ to the stochastic differential equation

$$
d x=\gamma(x) d t+\sigma(x) d W(t)
$$

where $W(t)$ ranges over a finite dimensional vector space $Y \subset X$, the drift $\gamma: X \mapsto X$ and the diffusion $\sigma: X \mapsto \mathcal{L}(Y, X)$ are smooth and bounded maps. In other words, it defines evolutions $t \mapsto \mathbb{X}(x, \omega)(t):=\mathbb{X}_{\omega}^{x}(t) \in X$ starting at $x$ at time 0 and parameterized by random events $\omega \in \Omega$ satisfying technical requirements (measurability, filtration, etc.) that are not relevant to involve at this stage of the exposition. The initial state $x$ being fixed, the random variable $\omega \mapsto \mathbb{X}(x, \omega):=\mathbb{X}_{\omega}^{x}(\cdot) \in \mathcal{C}(0, \infty ; X)$ is called a stochastic process.

When a subset $\mathcal{H} \subset \mathcal{C}(0, \infty ; X)$ of prescribed evolutions is a closed subset, we denote by $\mathbb{P}_{\mathbb{X}(x, \cdot)}$ the law of the random variable $\mathbb{X}(x, \cdot)$ defined by

$$
\mathbb{P}_{\mathbb{X}(x, \cdot)}(\mathcal{H}):=\mathbb{P}(\{\omega \mid \mathbb{X}(x, \omega) \in \mathcal{H}\})
$$

It is natural to introduce the stochastic core of $\mathcal{H}$ under the stochastic system: It is the subset of initial states $x$ from which starts a stochastic process $\omega \mapsto \mathbb{X}(x, \omega)$ such that for almost all $\omega \in \Omega, \mathbb{X}(x, \omega) \in \mathcal{H}$ :

$$
\operatorname{Stoc}_{\mathbb{X}}(\mathcal{H}):=\left\{x \in X \mid \text { for almost all } \omega \in \Omega, \mathbb{X}(x, \omega):=\mathbb{X}_{\omega}^{x}(\cdot) \in \mathcal{H}\right\}
$$

On the other hand, let us associate with drift and the diffusion the Stratonovitch drift $\widehat{\gamma}$ defined by $\widehat{\gamma}(x):=\gamma(x)-\frac{1}{2} \sigma^{\prime}(x) \sigma(x)$.

We associate with this stochastic differential equation the specific tychastic system

$$
\begin{cases}(i) & x^{\prime}(t)=\widehat{\gamma}(x(t))+\sigma(x(t)) v(t) \\ (i i) & v(t) \in Y\end{cases}
$$

where the tychastic map is constant and equal to $Y$. Denoting by $\mathcal{S}$ the evolutionary system associated with tychastic system 22 , we can associate with $\mathcal{H}$ its tychastic core defined by the subset

$$
\mathcal{S}^{\ominus 1}(\mathcal{H}):=\{x \in X \mid \mathcal{S}(x) \subset \mathcal{H}\}
$$


of initial states from which all evolutions governed by 22 belong to $\mathcal{H}$.

The definitions of the tychastic and stochastic cores of subsets of evolution properties are similar in spirit.

But there is a deeper similarity that we mention briefly: The Strook-Varadhan Support Theorem implies that whenever $\mathcal{H}$ is closed, the stochastic core of $\mathcal{H}$ under the stochastic system $\mathbb{X}$ and its tychastic core under the associated tychastic system $\mathcal{S}$ coincide:

$$
\operatorname{Stoc}_{\mathbb{X}}(\mathcal{H})=\mathcal{S}^{\ominus 1}(\mathcal{H})
$$

Taking for subset $\mathcal{H}$ the closed subset of evolutions viable in a closed environment $K$ forever or until it captures a closed target $C \subset K$, we deduce that the stochastic viability kernel with target under stochastic differential equation (19) is equal to the invariance kernel with target under the associated tychastic system (22).

Furthermore, the tychastic system associated with a stochastic one by the Strook-Varadhan Support Theorem is very particular: there is no bound on the tyches, whereas general tychastic systems allow the tyches to range over subsets $V(x)$ depending upon the state $x$, describing so to speak a state-dependent "tychastic volatility" (called "versatility"). This state-dependent uncertainty, unfortunately absent in the mathematical representation of uncertainty in the framework of stochastic processes, is of utmost importance for describing uncertainty in problems dealing with living beings.

\section{Palicinesia Functions}

We integrate now the definition of the environment $K$ in the definition of the tychastic map $V$ by setting $V(x)=\emptyset$ whenever $x \notin K$. We denote by $\mathcal{Q}(x, v)$ the set of solutions $(x(\cdot), v(\cdot))$ to the above tychastic system (15) starting at $(x, v)$.

Definition 8.1 The palicinesia $\sup _{t \geq 0}\left\|v^{\prime}(t)\right\|$ of a tyche is its maximal velocity over time.

The palicinesia function is the maximal palicinesia threshold under which all solutions $(x(\cdot), v(\cdot))$ to differential equation $x^{\prime}(t)=f(x(t), v(t))$ starting at $(x, v)$ satisfy the tychastic constraint $v(t) \in V(x(t))$.

In this section, we shall assume that $f$ is Lipschitz and that the graph of $V$ is closed.

\subsection{Viability Characterization of Palicinesia Functions}


We shall characterize the palicinesia function in terms of the invariance kernel of $\operatorname{Graph}(V) \times$ $\mathbb{R}_{+}$under the auxiliary system

$$
\begin{cases}(i) & x^{\prime}(t)=f(x(t), v(t)) \\ (i i) & v^{\prime}(t)=v_{1}(t) \\ (\text { iii }) & y^{\prime}(t)=0 \\ & \text { where }\left\|v_{1}(t)\right\| \leq y(t)\end{cases}
$$

Theorem 8.2 The palicinesia function is related to the invariance kernel of $\operatorname{Graph}(V) \times \mathbb{R}_{+}$ under system (24) by formula

$$
\beta(x, v)=\sup _{(x, v, y) \in \operatorname{Inv}_{24}\left(\operatorname{Graph}(V) \times \mathbb{R}_{+}\right)} y
$$

Proof - Indeed, to say that $(x, v, y)$ belongs to $\operatorname{Inv} \sqrt{24}\left(\operatorname{Graph}(V) \times \mathbb{R}_{+}\right)$amounts to saying that all evolutions $t \mapsto(x(t), v(t), y)$ governed by (24) are viable in $\operatorname{Graph}(V) \times \mathbb{R}_{+}$. In other words, all evolutions $(x(\cdot), v(\cdot))$ governed by $x^{\prime}(t)=f(x(t), v(t))$ satisfying

$$
\forall t \geq 0,\left\|v^{\prime}(t)\right\| \leq y
$$

are viable in $\operatorname{Graph}(V)$. This amounts to saying that $y \leq \beta(x, v)$.

Theorem 8.3 If $f$ is Lipschitz and the graph of $V$ is closed, the palicinesia function $\beta$ is upper semicontinuous.

Proof - Since the auxiliary system 24 is Lipschitz because $f$ is Lipschitz and because $\operatorname{Graph}(V) \times \mathbb{R}_{+}$is closed by assumption, then the invariance kernel $\operatorname{Inv} \sqrt{24}\left(\operatorname{Graph}(V) \times \mathbb{R}_{+}\right)$ is also closed. Then we deduce that the invariance kernel is the hypograph of the palicinesia function, which is therefore upper semicontinuous,

\subsection{Hamilton-Jacobi Equations for Palicinesia Functions}

One can prove that the palicinesia function $\beta$ is the largest nonnegative upper semicontinuous solution to the Hamilton-Jacobi-Bellman partial differential equation: $\forall(x, v) \in$ $\operatorname{Graph}(V)$,

$$
\left\langle\frac{\partial \mathbf{v}(x, v)}{\partial x}, f(x, v)\right\rangle-\mathbf{v}(x, v)\left\|\frac{\partial \mathbf{v}(x, v)}{\partial v}\right\|_{\star}=\sum_{i=1}^{n} \frac{\partial \mathbf{v}(x, v)}{\partial x_{i}} f_{i}(x, v)-\mathbf{v}(x, v)\left\|\frac{\partial \mathbf{v}(x, v)}{\partial v}\right\|_{\star} \geq 0
$$

on the graph of $V$, where $\|p\|_{\star}:=\sup _{\|v\| \leq 1}\|v\|$ denotes the dual norm (in an adequate generalized sense). 
To give a meaning to such generalized solutions, we introduce the (contingent) hypoderivative $D_{\downarrow} \beta(x, v)$ of $\beta$ at $(x, v) \in \operatorname{Dom}(\beta)$ defined by

$$
D_{\downarrow} \beta(x, v)\left(x^{\prime}, v^{\prime}\right):=\liminf _{h \rightarrow 0+, \xi \rightarrow x^{\prime}, \nu \rightarrow v^{\prime}} \frac{\beta(x+h \xi, v+h \nu)-\beta(x, v)}{h}
$$

We recall that the hypographs of these hypoderivatives are related to the contingent cones to the hypograph of the function by the following formulas:

$$
\mathcal{H} y p\left(D_{\downarrow} \beta(x, v)\right)=T_{\mathcal{H} y p(\beta)}(x, u, \beta(x, v))
$$

Theorem 8.4 Assume that the palicinesia function is continuous on its domain. Then it is the largest nonnegative upper semicontinuous fonction $\mathbf{v}$ defined on $\operatorname{Graph}(V)$ solution to

$$
\forall(x, v) \in \operatorname{Dom}(\mathbf{v}), \quad \inf _{\left\|v^{\prime}\right\| \leq \mathbf{v}(x, v)} D_{\downarrow} \mathbf{v}(x, v)\left(f(x, v), v^{\prime}\right) \geq 0
$$

Proof - This a consequence of Theorem 6.3 when $K:=\operatorname{Graph}(V) \times \mathbb{R}_{+}, C:=\emptyset$ and the tychastic system is auxiliary system 24.

It states that the hypograph $\mathcal{H} y p(\beta)$ of the palicinesia function, which is the invariance kernel of $\operatorname{Graph}(V) \times \mathbb{R}_{+}$under system (24) thanks to Theorem 6.3, is the largest closed subset $\mathcal{V}:=\mathcal{H} y p(\mathbf{v})$ of $\operatorname{Graph}(V) \times \mathbb{R}_{+}$such that for every $(x, u, c) \in \mathcal{V}:=\mathcal{H} y p(\mathbf{v})$,

$$
\forall\left\|v_{1}\right\| \leq \mathbf{v}(x, v), \quad\left(f(x, v), v_{1}, 0\right) \in T_{\mathcal{H} y p(\mathbf{v})}(x, u, c)
$$

If $c=\mathbf{v}(x, v)$, we deduce from formula

$$
T_{\mathcal{H} y p(\mathbf{v})}(x, v, \mathbf{v}(x, v))=\mathcal{H} y p\left(D_{\downarrow} \mathbf{v}(x, v)\right)
$$

that

$$
\forall\left\|v_{1}\right\| \leq \mathbf{v}(x, v), \quad D_{\downarrow} \mathbf{v}(x, v)\left(f(x, v), v_{1}\right) \geq 0
$$

This is equivalent to writing that

$$
\forall(x, v) \in \operatorname{Dom}(\mathbf{v}), \quad \inf _{\left\|v_{1}\right\| \leq \mathbf{v}(x, v)} D_{\downarrow} \mathbf{v}(x, v)\left(f(x, v), v_{1}\right) \geq 0
$$

This concludes the proof.

\section{$9 \quad$ Guaranteed Viability Kernels under Dynamical Games}

We summarize the main results on guaranteed viability/capturability of a target under dynamical games that we need to prove the results announced in the preceding section. 
We denote by $X, \mathcal{U}$ and $\mathcal{V}$ three finite dimensional vector spaces, and we introduce a continuous single-valued map $f: X \times \mathcal{U} \times \mathcal{V} \leadsto X$, a cybernetic set-valued map $U: X \leadsto \mathcal{U}$ and a tychastic set-valued map $V: X \leadsto \mathcal{V}$.

We consider a dynamical game described by

$$
\begin{cases}(i) & x^{\prime}(t)=f(x(t), u(t), v(t)) \\ (i i) & u(t) \in U(x(t)) \\ (i i i) & v(t) \in V(x(t))\end{cases}
$$

which is, so to speak, a control system regulated by two parameters, $u(t)$ and $v(t)$, the first one regarded as a regulating parameter, controlled by a player, the second one regarded as a tyche, chosen in a unknown way by "Nature".

We introduce a class $\widetilde{\mathcal{U}}$ of Lipschitz selections $x \mapsto \widetilde{u}(x) \in U(x)$, that are used as feedbacks or strategies by the player controlling the parameter $u$.

We associate with such a feedback $\widetilde{u}(x) \in U(x)$ the set $\mathcal{C}_{\widetilde{u}}(x)$ of solutions $(x(\cdot), v(\cdot)) \in$ $\mathcal{C}(0, \infty ; X) \times L^{1}(0, \infty ; \mathcal{V})$ to the parameterized system

$$
\begin{cases}(i) & x^{\prime}(t)=f(x(t), \widetilde{u}(x(t)), v(t)) \\ (i i) & v(t) \in V(x(t))\end{cases}
$$

starting at $x$.

We may identify the above dynamical game with the set-valued map $(x, \widetilde{u}) \leadsto \mathcal{C}_{\widetilde{u}}(x)$, that we regard as an evolutionary game.

Definition 9.1 Let $C \subset K \subset X$ be two subsets, $C$ being regarded as a target, $K$ as a constrained set.

We denote by $\operatorname{Inv}_{\widetilde{u}}(K, C)$ the invariance basin of $C$ in $K$ under tychastic system.

The subset

$$
\left[\operatorname{Viab}_{\widetilde{\mathcal{U}}} \operatorname{Inv}_{V}\right](K, C):=\bigcup_{\widetilde{u} \in \widetilde{\mathcal{U}}} \operatorname{Inv}_{\widetilde{u}}(K, C)
$$

of elements $x \in K$ such that there exists a feedback $\widetilde{u} \in \widetilde{\mathcal{U}}$ such that for every solutions $(x(\cdot), v(\cdot)) \in \mathcal{C}_{\widetilde{u}}(x), x(\cdot)$ is viable in $K$ forever or until a finite time $t^{*} \in \mathbf{R}_{+}$satisfying the viability/capturability conditions

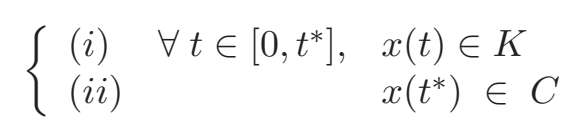

is called the guaranteed viable-capture basin of a target under the evolutionary game $(x, \widetilde{u}) \leadsto$ $\mathcal{C}_{\widetilde{v}}(x)$ defined on $X \times \widetilde{\mathcal{U}}$ (that, naturally, depends upon the choice of the family $\widetilde{\mathcal{U}}$ of feedbacks).

We deduce from [9, Aubin \& Catté] that 
Theorem 9.2 The guaranteed viability kernel $\left[\mathrm{Viab}_{\widetilde{\mathcal{u}}} \operatorname{Inv}_{V}\right](K, C)$ is the largest fixed point between $C$ and $K$ of the map $D \mapsto\left[\operatorname{Viab}_{\widetilde{\mathcal{U}}} \operatorname{Inv}_{V}\right](D, C)$.

Consequently, the guaranteed viability kernel satisfies

$$
\left[\operatorname{Viab}_{\widetilde{\mathcal{U}}} \operatorname{Inv}_{V}\right](K, C)=\left[\operatorname{Viab}_{\tilde{\mathcal{U}}} \operatorname{Inv}_{V}\right]\left(\left[\operatorname{Viab}_{\widetilde{\mathcal{U}}} \operatorname{Inv}_{V}\right](K, C), C\right)
$$

In other words, it is the largest subset of elements $x \in K$ such that there exists a feedback $\widetilde{u} \in \widetilde{\mathcal{U}}$ such that for every solutions $(x(\cdot), v(\cdot)) \in \mathcal{C}_{\widetilde{u}}(x)$, there exists $t^{*} \in \mathbf{R}_{+}$satisfying the viability/capturability conditions.

We shall assume that the dynamical game (25) is Lipschitz in the sense that the setvalued maps $U$ and $V$ are Lipschitz with compact values and that the single-valued map $f$ is Lipschitz with closed values.

Let $\widetilde{\mathcal{U}}_{\lambda}$ be the set of Lipschitz selections with constant $\lambda$ of the set-valued map $U$ : for every $x, \widetilde{u}(x) \in U(x)$.

The subset

$$
\left[\operatorname{Viab}_{\widetilde{\mathcal{U}}} \operatorname{Inv}_{V}\right](K, C):=\bigcup_{\widetilde{u} \in \widetilde{\mathcal{U}}_{\lambda}} \operatorname{Inv}_{\widetilde{u}}(K, C)
$$

is called the $\lambda$-guaranteed viable-capture basin of a target under the evolutionary game $(x, \widetilde{u}) \leadsto$ $\mathcal{C}_{\widetilde{u}}(x)$ associated with dynamical game 25 .

One can prove that when the game is Lipschitz, the set-valued map $(x, \widetilde{u}) \in X \times \widetilde{\mathcal{U}}_{\lambda} \leadsto$ $\mathcal{C}_{\widetilde{u}}(x) \subset \mathcal{C}(0, \infty ; X)$ is lower semicontinuous and consequently, that the $\lambda$-guaranteed viablecapture basin is closed.

Using the Viability and the Invariance Theorems, one can prove the following tangential properties of guaranteed viability kernels with targets:

Theorem 9.3 Let us assume that the dynamical game $(U, V, f)$ is Lipschitz, that $C \subset K$ and $K$ are closed subsets of $X$ and that $K \backslash C$ is a repeller under all the maps $(x, \widetilde{u}) \sim \mathcal{C}_{\widetilde{u}}(x)$.

Then the guaranteed viability kernel of target $C$ viable in $K$ is the largest of the closed subsets $D$ satisfying $C \subset D \subset K$ and

1. the tangential property

$$
\forall x \in D \backslash C, \exists u \in U(x) \text { such that } \forall v \in V(x), \quad f(x, u, v) \in T_{D}(x)
$$

\footnotetext{
${ }^{1}$ or, the equivalent dual formulation,$$
\forall x \in D \backslash C, \forall p \in N_{D}(x), \inf _{u \in U(x)} \sup _{v \in V(x)}\langle f(x, u, v), p\rangle \leq 0
$$

where the (regular) normal cone $N_{D}(x):=T_{D}(x)^{-}$is the polar cone to the contingent cone $T_{D}(x)$.
} 
2. there exists a $\lambda$-Lipschitz selection of the guaranteed regulation map $\Gamma_{D}$ defined by

$$
\forall x \in D \backslash C, \Gamma_{D}(x):=\left\{u \in U(x) \mid f(x, u, V(x)) \subset T_{D}(x)\right\}
$$

This theorem is a restatement of Theorems 9.2.14 and 9.2.18 of [2, Aubin, Chapter 9].

\section{Guaranteed Inertia Functions}

Let us consider a tychastic control system

$$
\left\{\begin{array}{l}
(i) \\
(i i)(u(t), v(t)) \in W(x(t))
\end{array} x^{\prime}(t)=f(x(t), u(t), v(t))\right.
$$

where $W: X \leadsto \mathcal{U} \times \mathcal{V}$ (for instance, $W(x):=U(x) \times V(x)$ ).

Definition 10.1 Let us consider a family of feedbacks $\widetilde{\mathcal{U}_{1}}$. The associated guaranteed inertia function $\gamma(x, u, v)$ is the maximal palicinesia threshold $c$ such that there exists a feedback $\widetilde{u}_{1} \in \widetilde{\mathcal{U}_{1}}$ satisfying $\left\|\widetilde{u}_{1}\right\| \leq c$ under which all solutions $(x(\cdot), v(\cdot))$ to tychastic system

$$
\begin{cases}(i) & x^{\prime}(t)=f(x(t), u(t), v(t)) \\ (i i) & u^{\prime}(t)=\widetilde{u}_{1}(x(t)) \\ (\text { iii }) & \left\|v^{\prime}(t)\right\| \leq c\end{cases}
$$

satisfy the tychastic-control constraint $(u(t), v(t)) \in W(x(t))$.

In this section, we shall assume that $f$ is Lipschitz and that the graph of $W$ is closed.

\subsection{Viability Characterization of Guaranteed Inertia Functions}

We shall characterize the guaranteed inertia function in terms of the guaranteed viability kernel of $\operatorname{Graph}(W) \times \mathbb{R}_{+}$under the auxiliary dynamical game

$$
\begin{cases}(i) & x^{\prime}(t)=f(x(t), u(t), v(t)) \\ (\text { iii }) & u^{\prime}(t)=u_{1}(t) \\ (i i) & v^{\prime}(t)=v_{1}(t) \\ (\text { iii }) & y^{\prime}(t)=0 \\ & \text { where } \max \left(\left\|u_{1}(t)\right\|,\left\|v_{1}(t)\right\|\right) \leq y(t)\end{cases}
$$

Theorem 10.2 The guaranteed inertia function is related to the guaranteed viability kernel of $\operatorname{Graph}(W) \times \mathbb{R}_{+}$under auxiliary dynamical game 29) by formula

$$
\gamma(x, u, v)=\sup _{(x, u, v, y) \in\left[\operatorname{Viab}_{\widetilde{\mathcal{U}}_{1}} \operatorname{Inv}_{V_{1}}\right]_{[2]}\left(\operatorname{Graph}(W) \times \mathbb{R}_{+}\right)} y
$$


Proof - Indeed, to say that $(x, u, v, y)$ belongs to $\left[\operatorname{Viab}_{\widetilde{\mathcal{U}}_{1}} \operatorname{Inv}_{V_{1}}\right]_{[29 p}\left(\operatorname{Graph}(V) \times \mathbb{R}_{+}\right)$ amounts to saying that there exist a Lipschitz selection $\widetilde{u}_{1}$ satisfying $\left\|\widetilde{u}_{1}\right\| \leq y$ such that all evolutions $t \mapsto(x(t), u(t), v(t), y)$ governed by

$$
\begin{cases}(i) & x^{\prime}(t)=f(x(t), u(t), v(t)) \\ (i i i) & u^{\prime}(t)=\widetilde{u}_{1}(x(t)) \\ (i i) & v^{\prime}(t)=v_{1}(t) \\ (\text { iii }) & y^{\prime}(t)=0\end{cases}
$$

are viable in $\operatorname{Graph}(V) \times \mathbb{R}_{+}$. In other words, all solutions $(x(\cdot), v(\cdot))$ governed by $x^{\prime}(t)=$ $f(x(t), u(t), v(t)), u^{\prime}(t)=\widetilde{u}_{1}(x(t))$ and satisfying

$$
\forall t \geq 0,\left\|v^{\prime}(t)\right\| \leq y
$$

are viable in $\operatorname{Graph}(W)$. This amounts to saying that $y \leq \gamma(x, u, v)$.

\subsection{Hamilton-Jacobi Equations for Guaranteed Inertia Functions}

One can prove that the guaranteed inertia function $\gamma$ is the largest nonnegative upper semicontinuous solution to the Hamilton-Jacobi-Isaacs partial differential equation: $\forall(x, u, v) \in$ $\operatorname{Graph}(W)$,

$$
\left\langle\frac{\partial \mathbf{w}(x, u, v)}{\partial x}, f(x, v)\right\rangle+\mathbf{w}(x, u, v)\left\|\frac{\partial \mathbf{w}(x, u, v)}{\partial u}\right\|_{\star}-\mathbf{w}(x, u, v)\left\|\frac{\partial \mathbf{w}(x, u, v)}{\partial v}\right\|_{\star}=0
$$

on the graph of $V$, where $\|p\|_{\star}:=\sup _{\|v\| \leq 1}\langle p, v\rangle$ denotes the dual norm.

Theorem 10.3 Assume that the guaranteed inertia function function is continuous on its domain. Then it is the largest nonnegative upper semicontinuous fonction $\mathbf{w}$ defined on $\operatorname{Graph}(W)$ solution to

$$
\forall(x, u, v) \in \operatorname{Dom}(\mathbf{w}), \quad \sup _{\left\|u_{1}\right\| \leq \mathbf{w}(x, u, v)} \inf _{\left\|v_{1}\right\| \leq \mathbf{w}(x, u, v)} D_{\downarrow} \mathbf{w}(x, u, v)\left(f\left(x, u, u_{1}, v_{1}\right) \geq 0\right.
$$

and satisfying the property

$$
\inf _{\left\|v_{1}\right\| \leq \mathbf{w}(x, v)} D_{\downarrow} \mathbf{w}(x, v)\left(f\left(x, v, v^{\prime}\right) \geq 0\right.
$$

Proof - This a consequence of Theorem 9.3 when $K:=\operatorname{Graph}(W) \times \mathbb{R}_{+}, C:=\emptyset$ and the tychastic system is auxiliary system 29 .

It states that the hypograph $\mathcal{H} y p(\gamma)$ of the guaranteed inertia function, which is the guaranteed viability kernel of $\operatorname{Graph}(W) \times \mathbb{R}_{+}$under the dynamical game (29) thanks to 
Theorem 9.3, is the largest closed subset $\mathcal{V}:=\mathcal{H} y p(\mathbf{w})$ of $\operatorname{Graph}(W) \times \mathbb{R}_{+}$such that for every $(x, u, v, c) \in \mathcal{V}:=\mathcal{H} y p(\mathbf{w})$,

$\exists\left\|u_{1}\right\| \leq \mathbf{w}(x, v)$ such that $\forall\left\|v_{1}\right\| \leq \mathbf{w}(x, v), \quad\left(f(x, u, v), u_{1}, v_{1}, 0\right) \in T_{\mathcal{H}_{y p(\mathbf{w})}}(x, u, v, c)$

If $c=\mathbf{w}(x, u, v)$, we deduce from formula

$$
T_{\mathcal{H} y p(\mathbf{w})}(x, u, v, \mathbf{w}(x, u, v))=\mathcal{H} y p\left(D_{\downarrow} \mathbf{w}(x, u, v)\right)
$$

that

$$
\exists\left\|u_{1}\right\| \leq \mathbf{w}(x, u, v) \text { such that } \forall\left\|v_{1}\right\| \leq \mathbf{w}(x, u, v), \quad D_{\downarrow} \mathbf{w}(x, u, v)\left(f(x, u, v), u_{1}, v_{1}\right) \geq 0
$$

This is equivalent to writing that

$$
\forall(x, u, v) \in \operatorname{Dom}(\mathbf{w}), \quad \inf _{\left\|v_{1}\right\| \leq \mathbf{w}(x, u, v)} D_{\downarrow} \mathbf{w}(x, u, v)\left(f(x, u, v), u_{1}, v_{1}\right) \geq 0
$$

This concludes the proof. 


\section{References}

[1] AUBIN J.-P. (1991) Viability Theory Birkhäuser, Boston, Basel, Berlin

[2] AUBIN J.-P. (1997) Dynamic Economic Theory: A Viability Approach, SpringerVerlag

[3] AUBIN J.-P. (1999) Impulse Differential Inclusions and Hybrid Systems: A Viability Approach, Lecture Notes, University of California at Berkeley

[4] AUBIN J.-P. (2001) Viability Kernels and Capture Basins of Sets under Differential Inclusions, SIAM J. Control, 40, 853-881

[5] AUBIN J.-P. (to appear) Boundary-Value Problems for Systems of Hamilton-JacobiBellman Inclusions with Constraints, SIAM J. Control, 41, 425-456

[6] AUBIN J.-P., BAYEN A., BONNEUIL N. \& SAINT-PIERRE P. (in preparation) Viability, Control and Games: Regulation of Complex Evolutionary Systems Under Uncertainty and Viability Constraints

[7] AUBIn J.-P., BERNARDO T. \& SAINT-PIERRE P. (2004) A Viability Approach to Global Climate Change Issues, Advances in Global Change Research, Haurie A \& Viguier (Eds.), Klüwer

[8] AUBIN J.-P., BERNARDO T. \& SAINT-PIERRE P. (2005) Inertia Functions and Heavy Evolutions, preprint

[9] AUBIN J.-P. \& CATTE F. (2001) Fixed-Point and Algebraic Properties of Viability Kernels and Capture Basins of Sets, 10, 379-416

[10] AUBIN J.-P. \& DORDAN O. (1996) Fuzzy Systems, Viability Theory and Toll Sets, In Handbook of Fuzzy Systems, Modeling and Control, Hung Nguyen Ed.. Kluwer, 461-488

[11] AUBIN J.-P. \& FRANKOWSKA H. (1985) Heavy viable trajectories of controlled systems, Proceedings of Dynamics of Macrosystems, IIASA, September 1984,, Ed. Aubin J.-P., Saari D.\& Sigmund K., Springer-Verlag,148-167

[12] AUBIN J.-P. \& FRANKOWSKA H. (1990) Set-Valued Analysis, Birkhäuser

[13] BARRON E.N. \& JENSEN R. (1990) Semicontinuous viscosity solutions for HamiltonJacobi equations with convex Hamiltonians, Comm. Partial Differential Equations, 15, 1713-1742

[14] CARDAliagueT P. (1994) Domaines dicriminants en jeux différentiels, Thèse de l'Université de Paris-Dauphine

[15] CARDALIAGUET P. (1996) A differential game with two players and one target, SIAM J. Control and Optimization, 34, 4, 1441-1460. 
[16] CARDALIAGUET P. (1997) On the regularity of semi-permeable surfaces in control theory with application to the optimal exit-time problem (Part II), S.I.A.M. Journal on Opt. Control and Opti.,

[17] CARDALIAGUET P. (2000) Introduction à la théorie des jeux différentiels, Lecture Notes, Université Paris-Dauphine

[18] CARDALIAGUET P., QUINCAMPOIX M. \& SAINT-PIERRE P. (1995) Contribution à l'étude des jeux différentiels quantitatifs et qualitatifs avec contrainte sur l'état, ComptesRendus de l'Académie des Sciences, 321, 1543-1548

[19] CARDAliagUET P., QUINCAMPOIX M. \& SAINT-PIERRE P. (1999) Set-valued numerical methods for optimal control and differential games, In Stochastic and differential games. Theory and numerical methods, Annals of the International Society of Dynamical Games, 177-247 Birkhäuser

[20] CURY P., MULLON C., GARCIA S. \& SHANNON L. (2005) Viability theory for an ecosystem approach to fisheries, ICES Journal of Marine Science, 62, 577-584

[21] CURY P., MULLON C. \& SHANNON L. (2004) Viability Model of trophic Interactions in marine ecosystems, Natural Resource Modeling 17, 27-58

[22] FRANKOWSKA H. (1987) L'équation d'Hamilton-Jacobi contingente, Comptes-Rendus de l'Académie des Sciences, PARIS, Série 1, 304, 295-298

[23] FRANKOWSKA H. (1987) Optimal trajectories associated to a solution of contingent Hamilton-Jacobi equations, IEEE, 26th, CDC Conference, Los Angeles, December 9-11

[24] FRANKOWSKA H. (1989) Optimal trajectories associated to a solution of contingent Hamilton-Jacobi equations, Applied Mathematics and Optimization, 19, 291-311

[25] FRANKOWSKA H. (1989) Hamilton-Jacobi equation: viscosity solutions and generalized gradients, J. of Math. Analysis and Appl. 141, 21-26

[26] FRANKOWSKA H. (1991) Lower semicontinuous solutions to Hamilton-Jacobi-Bellman equations, Proceedings of 30th CDC Conference, IEEE, Brighton, December 11-13

[27] FRANKOWSKA H. (1993) Lower semicontinuous solutions of Hamilton-Jacobi-Bellman equation, SIAM J. on Control and Optimization,

[28] PUJAL D. \& SAINT-PIERRE P. (2001) L'algorithme du bassin de capture appliqué pour évaluer des options européennes, américaines ou exotiques, preprint

[29] QUINCAMPOIX M. (1990) Frontières de domaines d'invariance et de viabilité pour des inclusions différentielles avec contraintes, Comptes-Rendus de l'Académie des Sciences, Paris, 311, 411-416

[30] QUINCAMPOIX M. (1990) Playable differentiable games, J. Math. Anal. Appl., 194-211

[31] QUINCAMPOIX M. (1992) Differential inclusions and target problems, SIAM J. Control and Optimization, 30, 324-335 
[32] QUINCAMPOIX M. (1992) Enveloppes d'invariance pour des inclusions différentielles Lipschitziennes : applications aux problèmes de cibles, Comptes-Rendus de l'Académie des Sciences, Paris, 314, 343-347

[33] QUINCAMPOIX M. (1990) Frontières de domaines d'invariance et de viabilité pour des inclusions différentielles avec contraintes, Comptes-Rendus de l'Académie des Sciences, Paris, 311, 411-416

[34] QUINCAMPOIX M. \& SAINT-PIERRE (1995) An algorithm for viability kernels in Hölderian case: Approximation by discrete viability kernels, J. Math. Syst. Est. and Control, Summary : 115-120

[35] QUINCAMPOIX M. \& VELIOV V. (1998) Viability with a target: theory and applications, in Applications of mathematics in engineering, 47-54, Heron Press

[36] ROCKAFELLAR R.T. \& WETS R. (1997) Variational Analysis, Springer-Verlag

[37] SAINT-PIERRE P. (1994) Approximation of the viability kernel, Applied Mathematics \& Optimisation, 29, 187-209

[38] SAINT-PIERRE P. (2002) Hybrid kernels and capture basins for impulse constrained systems, in Hyrid Systems: Computation and Controls, Tomlin C. and Greenstreet M,. Eds, Springer Verlag, 378-392 


\section{Contents}

\begin{tabular}{llr}
\hline 1 & Introduction & 2
\end{tabular}

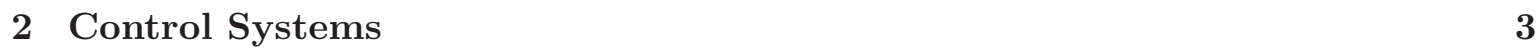

\begin{tabular}{lll}
\hline 3 & Inertia Functions & 6
\end{tabular}

3.1 Viability Characterization of Inertia Functions $\ldots \ldots \ldots$. . . . . . . . . . . .

3.2 Hamilton-Jacobi Equations for Inertia Functions . . . . . . . . . . . . . 8

3.3 Behavior of Heavy Evolutions . . . . . . . . . . . . . . . . . . . . . . 11

4 Example: Evolution of the Biomass of a Renewable Resource 12

4.1 From Malthus to Verhulst and Beyond . . . . . . . . . . . . . . . . . . . . 12

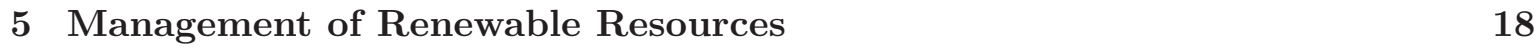

5.1 Inert Evolutions . . . . . . . . . . . . . . . . . . . . 23

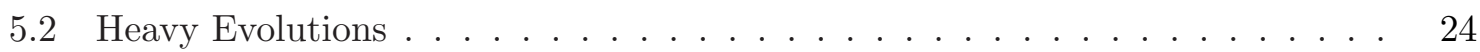

5.3 Towards Dynamical Games $\ldots \ldots \ldots \ldots$. . . . . . . . . . . . . . . 25

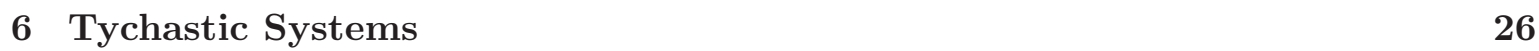

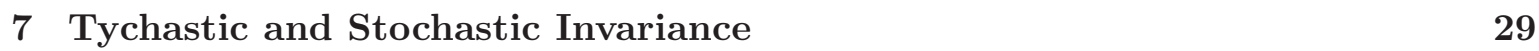

\begin{tabular}{lll}
\hline & Palicinesia Functions & 30
\end{tabular}

8.1 Viability Characterization of Palicinesia Functions . . . . . . . . . . . . 30

8.2 Hamilton-Jacobi Equations for Palicinesia Functions . . . . . . . . . . . . . . 31

9 Guaranteed Viability Kernels under Dynamical Games 32

10 Guaranteed Inertia Functions

10.1 Viability Characterization of Guaranteed Inertia Functions . . . . . . . . . . 35

10.2 Hamilton-Jacobi Equations for Guaranteed Inertia Functions . . . . . . . . . 36 